\title{
Effects of Clonal Reproduction on Evolutionary Lag and Evolutionary Rescue
}

\author{
Maria E. Orive, ${ }^{1, \star}$ Michael Barfield, ${ }^{2}$ Carlos Fernandez, ${ }^{1}$ and Robert D. Holt ${ }^{2}$ \\ 1. Department of Ecology and Evolutionary Biology, University of Kansas, Lawrence, Kansas 66045; 2. Department of Biology, \\ University of Florida, Gainesville, Florida 32611 \\ Submitted September 28, 2016; Accepted April 10, 2017; Electronically published August 11, 2017 \\ Online enhancements: appendix with three parts.
}

\begin{abstract}
AвSTRACт: Evolutionary lag - the difference between mean and optimal phenotype in the current environment-is of keen interest in light of rapid environmental change. Many ecologically important organisms have life histories that include stage structure and both sexual and clonal reproduction, yet how stage structure and clonality interplay to govern a population's rate of evolution and evolutionary lag is unknown. Effects of clonal reproduction on mean phenotype partition into two portions: one that is phenotype dependent, and another that is genotype dependent. This partitioning is governed by the association between the nonadditive genetic plus random environmental component of phenotype of clonal offspring and their parents. While clonality slows phenotypic evolution toward an optimum, it can dramatically increase population survival after a sudden step change in optimal phenotype. Increased adult survival slows phenotypic evolution but facilitates population survival after a step change; this positive effect can, however, be lost given survival-fecundity trade-offs. Simulations indicate that the benefits of increased clonality under environmental change greatly depend on the nature of that change: increasing population persistence under a step change while decreasing population persistence under a continuous linear change requiring de novo variation. The impact of clonality on the probability of persistence for species in a changing world is thus inexorably linked to the temporal texture of the change they experience.
\end{abstract}

Keywords: clonal reproduction, evolutionary lag, evolutionary rescue, stage structure.

\section{Introduction}

In a rapidly changing environment, organisms must constantly evolve to maintain fitness and persist. Rapid change may be inflicted by coevolving pathogens or other competing species, changes in temperature or other climatic condi-

\footnotetext{
* Corresponding author; e-mail: morive@ku.edu. ORCIDs: Orive, http://orcid.org/0000-0003-4825-0554
}

Am. Nat. 2017. Vol. 190, pp. 469-490. (C) 2017 by The University of Chicago 0003-0147/2017/19004-57297\$15.00. All rights reserved. This work is licensed under a Creative Commons Attribution 4.0 International License (CC BY 4.0), which permits reuse of the work with attribution. DOI: $10.1086 / 693006$ tions, or human impacts (e.g., release of toxicants). Evolution takes time- both for favorable new variants to arise and for those variants to increase in frequency and become fixed in a population. Following environmental change, populations may experience transient periods with a degree of maladaptation, reflecting the magnitude of that change, plastic responses, and the scope for rapid evolution. The relative difference between the current mean fitness of a population and the mean fitness of a hypothetical population fixed for all favorable genotypes is termed "evolutionary lag" (or "lag load"; see Maynard Smith 1976a, 1976b); evolutionary lag for a quantitative trait can also denote the difference between the mean phenotype and the optimal phenotype for a particular trait, a measure of maladaptation (Crespi 2000). When the evolutionary lag is large, species may be so maladapted as to be doomed to extinction-a scenario that has doubtless occurred often in the history of life and one that is of growing concern in the current biodiversity crisis (Wilson 2016). In contrast, if species adapt to novel circumstances quickly, they may escape extinction via evolutionary adaptation (evolutionary rescue; for a review, see Bell 2013) and exhibit either a population size recovery from an initial decline (Gomulkiewicz and Holt 1995) or more cryptic eco-evolutionary dynamics where ongoing adaptation prevents a dramatic decline under continuous environmental change (Kinnison et al. 2015).

The theme of evolutionary rescue is receiving increasing theoretical and empirical attention (e.g., Gonzalez and Bell 2013; Gonzalez et al. 2013; Martin et al. 2013; Schiffer et al. 2013; Uecker et al. 2014). Current projections of global environmental change, such as climate change, land use transformation, and the global movement of exotic species, make it likely that many species will increasingly experience maladaptation and evolutionary lags. While plastic phenotypic responses and dispersal may moderate the demographic impact of evolutionary lag in the short term, the long-term effects of these rapid changes will likely involve evolution and even extinction (Holt 1990; for a review, see Hoffmann and Sgrò 2011; for a discussion of the possible role played 
by evolution in coral reef persistence, see Pandolfi et al. 2011). Characterizing how genetic architecture and life-history structure affect evolutionary rates is crucial for understanding and predicting patterns of evolution and extinction in our rapidly changing world.

Many ecologically important organisms, such as reefbuilding corals and key plant species, including perennial grasses in prairies and savannahs, have populations characterized by complex life histories. These life histories may include modular organization, indeterminate growth leading to stage-structured populations, and both asexual (e.g., clonal) and sexual reproduction. Despite the ecological importance of such species, how stage structure and clonality interact to govern the rate of evolution and amount of evolutionary lag after environmental change is poorly understood. In this article, we address how the joint effects of clonal reproduction and stage structure can influence evolutionary lag and rescue, focusing on the evolution of a phenotypic trait in a stage-structured population with a mixture of sexual and asexual reproduction.

Many factors contribute to the rate of evolution of a population and thus to its evolutionary lag. One key factor is population size, which obviously affects the amount of genetic variation available for selection to act on (from both the initial variation and the input of novel mutations; see Orr and Unckless 2008, 2014; Gandon et al. 2013) but also influences the amount of time available for evolutionary rescue (Gomulkiewicz and Holt 1995). A population may decrease in size due to lowered fitness after environmental change; a larger population has more time before it becomes small enough to be vulnerable to extinction due to demographic stochasticity, Allee effects, or inbreeding depression. The potential for evolutionary rescue is also closely tied to life-history details determining the amount of genetic variation maintained by the population and to the distribution of fitness across the population. Gomulkiewicz and Houle (2009) derive critical levels of heritability (and thus minimum amounts of additive genetic variance) necessary for evolutionary rescue under either a single shift or constant change in the phenotypic optimum. They consider different demographic criteria for rescue, from highly stringent (population size never declines) to less stringent (population size must stay above some quasi-extinction threshold size; see Gomulkiewicz and Holt 1995). When considering the effects of finite population size, it is important to consider the distribution of absolute fitness (rather than focusing only on relative fitness; Bell 2013). For a declining population to survive, at least some individuals must obtain an absolute fitness (expected number of offspring) greater than 1, thus necessitating a focus on the upper tail of the absolute fitness distribution.

These factors and others may limit adaptation via natural selection by determining how rapidly populations respond to selection or by imposing absolute limits on the magnitude of evolution (reviewed in Barton and Partridge 2000). A key component often overlooked when considering constraints on evolution is the effect of either obligate or facultative asexual reproduction. Clonal (asexual) reproduction can result in new demographic individuals (as opposed to new genetic individuals; see Orive 1993) that differ from sexually produced offspring in key ways, such as the incorporation of somatic mutations for clonal offspring formed from somatic tissues and how the phenotype of the offspring is influenced by nonadditive genetic factors determining the parent's phenotype. In addition, many clonal organisms have life histories better described by morphological or developmental stage classes rather than age classes (Jackson et al. 1985). For such stagestructured life histories, individuals in a particular stage class may contribute to any of several different classes (via growth, fragmentation, sexual reproduction, or clonal reproduction) or remain in the same class (indeterminate growth) over time.

To determine how clonal reproduction and stage structure jointly impact evolutionary lag and the probability of evolutionary rescue, we develop a general model for phenotypic evolution with both sexual and clonal reproduction. A framework for analyzing evolution in stage-structured populations was presented in Barfield et al. (2011). Although the framework presented there is general, specific equations were derived assuming only sexual reproduction, and some derivations moreover assumed that for offspring born into each stage, mean phenotype matches mean genotype. This framework needs to be extended in order to examine how differences between sexual and clonal offspring may influence evolutionary lag and rescue.

Here, we explicitly allow both sexual and clonal reproduction and consider both the incorporation of somatic mutations into offspring produced by clonal reproduction and the possibility that clonal offspring may inherit not only the parental genotype (the additive genetic part of the phenotype) but also some or all of the nonadditive genetic and random environmental component of the parental phenotype. As concrete illustrations, we analytically explore simple one- and two-stage life histories that include both sexual and clonal reproduction. These life histories then form the basis for individual-based simulations of two contrasting scenarios of environmental change and evolutionary lag: a one-step change in the optimal phenotype and a linear, continuous change in the optimum. We show that the response of clonal organisms to rapid environmental change depends both on the magnitude of the change and on whether the population experiences that change as a single step or as continual change. We find that clonal reproduction in some circumstances facilitates evolutionary rescue, while in others it leads to a greater risk of population extinction. 


\section{A General Model for Evolution in Stage-Structured Populations with Clonal Reproduction}

We previously developed a general deterministic model for evolution in stage-structured populations assuming sexual reproduction (Barfield et al. 2011, 2014). We here (and in app. A, pt. A, and app. B, pt. A; app. B is available online) extend these results to include both sexual and clonal reproduction.

Individuals are assumed to have a multivariate quantitative trait (phenotype), $\mathbf{z}$, on which selection acts (because $\mathbf{z}$ affects fecundities and probabilities of transitions and death), where $\mathbf{z}=\left(z_{1}, z_{2}, \ldots, z_{m}\right)^{\mathrm{T}}, m$ is the number of components in $\mathbf{z}$, and $\mathrm{T}$ indicates the transpose. Let $\mathbf{z}=\mathbf{g}+\mathbf{e}$, where $\mathbf{g}$ is the additive genetic (breeding) value of the trait and $\mathbf{e}$ is the nonadditive genetic value plus the random environmental deviation (e.g., developmental noise). We model trait evolution over discrete time steps, deriving recursions for the joint probability density function (PDF) of genotype and phenotype. For stage $j$, this joint PDF in the current time step is denoted $p_{j}(\mathbf{g}, \mathbf{z})$, while that in the next time step is $p_{j}^{\prime}(\mathbf{g}, \mathbf{z})$. (Primes indicate the next time step, and $i$ and $j$ indicate stage; summations over $i$ or $j$ are over all stages, and integrals over $\mathbf{g}$ or $\mathbf{z}$ are over all values.) With this general machinery, we then find recursions for the mean genotype and phenotype of each stage assuming the joint PDFs are Gaussian. Detailed derivations of the recursions for both the number of individuals of each stage $i\left(N_{i}^{\prime}\right.$; eq. [A2]) and the joint genotypic and phenotypic PDF of each stage $i$ $\left(p_{i}^{\prime}(\mathbf{g}, \mathbf{z})\right.$; eq. [A3], with clonal contribution in eq. [A4]) are in appendix A, part A. Appendix A, part A, also derives results using specific assumptions about clonal reproduction, which are discussed below and used for later results.

To construct these recursions, we considered how clonal offspring may differ from sexual offspring, first considering their genotypes. In the absence of somatic mutation, the genotypic distribution of clonal offspring would match that of their parents. However, since some forms of clonal reproduction permit incorporation of somatic mutations into clonal offspring (Jackson et al. 1985; Orive 2001; Herrera 2009), we allow somatic mutation to change the distribution. One approach to modeling somatic mutation is as an additive value perturbing the genotype, so that offspring genotype is the sum of the parental genotype and a mutation effect with a zero-mean multivariate Gaussian distribution. The resulting distribution of clonal offspring genotypes is given in appendix A, part A (eq. [A5]).

We next considered how clonal offspring phenotype $\mathbf{z}$ depends on its own genotype $\mathbf{g}$ as well as on both its parental genotype $\mathbf{g}^{*}$ and phenotype $\mathbf{z}^{*}$. This could be the same as for sexual offspring (e.g., the phenotype could be the sum of a genotypic contribution and an independent zero-mean Gaussian random component e). However, some types of clonal reproduction may affect the mapping of $\mathbf{g}$ and $\mathbf{e}$ onto phenotype. For instance, if the environmental (random) effects on phenotype are fixed early in development and clonal reproduction involves later developmental stages, the environmental value of the clonal offspring phenotype may be correlated with the environmental value of the parent's phenotype, leading to systematic differences between clonal and sexual offspring (see "Discussion"). Therefore, we define an $m$-dimensional vector of association parameters, $\boldsymbol{\rho}_{i j}$, which gives the association between e of a stage $i$ clonal offspring and $\mathbf{e}^{*}$ of its stage $j$ parent. The quantity $\rho_{i j k}$ gives this association for the $k$ th component of the multivariate trait $\left(z_{k}\right)$. We assume that each offspring component of $\mathbf{e}$ is generated by taking a weighted sum of the corresponding parental component (with weight $\rho_{i j k}$ ) and a random portion (see eq. [A6]). If $\rho_{i j k}=0$, the offspring environmental value is completely randomly determined (as usually assumed for sexual reproduction). If $\rho_{i j k}=1$, the environmental value of the parent is passed on exactly to its clonal offspring. Other values of $\rho_{i j k}$ (between 0 and 1) result in the environmental part of clonal offspring phenotype being partly random and partly determined by the parental value. With this assumption for all components of $\mathbf{e}$, the PDF for clonal offspring phenotype given genotype $\left(Y_{i j}\left(\mathbf{z} \mid \mathbf{g}, \mathbf{g}^{*}, \mathbf{z}^{*}\right)\right)$ can be derived (see the last paragraph of app. A, pt. A).

Recursions for Mean Genotype and Mean Phenotype Assuming Gaussian Distributions. The general formalism in appendix A, part A, makes no particular assumptions about the distributions of genotypes and phenotypes. To make more explicit and detailed evolutionary predictions, genotypes and phenotypes are often assumed to have Gaussian distributions. This is often a reasonable approximation for polygenic traits and sexual reproduction, especially with no linkage. However, with increasing clonal reproduction, this assumption is likely to become less accurate. In appendix B, part B, we discuss this issue and present results comparing the use of analytical equations derived here by means of the Gaussian assumption with an individual-based simulation model (discussed below) that does not make this assumption; there is good agreement, except for high clonality and parent-offspring association (both 0.9). This suggests that the Gaussian assumption is often reasonable, even with clonality.

Following Barfield et al. (2014), we define, for each stage $i$, the vector of genotypes $\mathbf{g}_{i}$ (with mean $\overline{\mathbf{g}}_{i}$ ), the vector of phenotypes $\mathbf{z}_{i}$ (with mean $\overline{\mathbf{z}}_{i}$ ), and the vector of environmental contributions to the phenotype, $\mathbf{e}_{i}$. We assume that $\mathbf{g}_{i}$ and $\mathbf{z}_{i}$ are jointly Gaussian with additive-genetic covariance matrix $\mathbf{G}_{i}$ and phenotypic covariance matrix $\mathbf{P}_{i}$. We also assume that $\mathbf{z}_{i}=\mathbf{g}_{i}+\mathbf{e}_{i}$ and that $\mathbf{g}_{i}$ and $\mathbf{e}_{i}$ are independent (which means that the covariance between $\mathbf{g}_{i}$ and $\mathbf{z}_{i}$ is $\mathbf{G}_{i}$ ). Therefore, the distribution of the concatenation of $\mathbf{g}_{i}$ and $\mathbf{z}_{i}$ is nor- 
mally distributed with mean $\left(\overline{\mathbf{g}}_{i} \overline{\mathbf{z}}_{i}\right)^{\mathrm{T}}$ and variance-covariance matrix $\mathbf{M}$, which has $\mathbf{P}_{i}$ in the lower right quadrant and $\mathbf{G}_{i}$ in the other three quadrants (see Barfield et al. 2014).

We wish to model changes in the mean genotype $\left(\Delta \overline{\mathbf{g}}_{i}\right)$ and mean phenotype $\left(\Delta \overline{\mathbf{z}}_{i}\right)$ per time step, and so we need to find the mean genotype $\left(\overline{\mathbf{g}}_{i}^{\prime}\right)$ and phenotype $\left(\overline{\mathbf{z}}_{i}^{\prime}\right)$ for each stage $i$ in the next time step in terms of the mean genotype and phenotype of the current time step. Appendix B, part A, provides detailed derivations for both of these recursions. The recursion for mean genotype is

$$
\begin{aligned}
\overline{\mathbf{g}}_{i}^{\prime} & =\frac{1}{N_{i}^{\prime}} \sum_{j} N_{j} \int a_{i j}(\mathbf{z}) \int \mathbf{g} p_{j}(\mathbf{g}, \mathbf{z}) d \mathbf{g} d \mathbf{z} \\
& =\sum_{j} d_{i j} \overline{\mathbf{g}}_{j}+\sum_{j} d_{i j} \mathbf{G}_{j} \nabla_{\overline{\mathbf{z}}_{j}} \ln \bar{a}_{i j},
\end{aligned}
$$

where $a_{i j}(\mathbf{z})$ is the total contribution of a stage $j$ individual with phenotype $\mathbf{z}$ to stage $i$ (sum of direct transitions and sexual and clonal reproduction), $\bar{a}_{i j}$ is its average over the joint genotypic and phenotypic distribution of stage $j$ (see eqq. [A1]), and $d_{i j}=\bar{a}_{i j} N_{j} / N_{i}^{\prime}$ is the fraction of stage $i$ individuals in the next time step contributed by stage $j$. (We note here that $d_{i j}$ was written as $c_{i j}$ in Barfield et al. [2011].) As in Barfield et al. (2011), $\nabla_{\bar{z}_{i}}=\left(\partial / \partial \bar{z}_{1}, \partial / \partial \bar{z}_{2}, \ldots, \partial / \partial \bar{z}_{m}\right)^{\mathrm{T}}$ is the gradient operator with respect to trait means evaluated at $\overline{\mathbf{z}}_{j}$, the multivariate mean phenotype of stage $j$ individuals. The first sum of the last expression in (1) is a weighted average of stage-specific mean genotypes contributing to stage $i$, and the second sum gives the combined effects of selection.

For the recursion for mean phenotype, the derivation in appendix $\mathrm{B}$, part $\mathrm{A}$, gives

$$
\begin{aligned}
\overline{\mathbf{z}}_{i}^{\prime}= & \sum_{j}\left[\left(d_{i j}^{t}+\mathbf{R}_{i j} d_{i j}^{c}\right) \overline{\mathbf{z}}_{j}+\left(d_{i j}^{f}+\left(\mathbf{I}-\mathbf{R}_{i j}\right) d_{i j}^{c}\right) \overline{\mathbf{g}}_{j}\right] \\
& +\sum_{j}\left[d_{i j}^{t} \mathbf{P}_{j} \nabla_{\overline{\mathbf{z}}_{j}} \ln \bar{t}_{i j}+d_{i j}^{f} \mathbf{G}_{j} \nabla_{\overline{\mathbf{z}}_{j}} \ln \bar{f}_{i j}\right. \\
& \left.+\mathbf{R}_{i j} d_{i j}^{c} \mathbf{P}_{j} \nabla_{\overline{\mathbf{z}}_{j}} \ln \bar{c}_{i j}+\left(\mathbf{I}-\mathbf{R}_{i j}\right) d_{i j}^{c} \mathbf{G}_{j} \nabla_{\overline{\mathbf{z}}_{j}} \ln \bar{c}_{i j}\right],
\end{aligned}
$$

where $\bar{t}_{i j}, \bar{f}_{i j}$, and $\bar{c}_{i j}$ (eqq. [A1]) are the mean contribution to stage $i$ by a stage $j$ individual by direct transition, sexual reproduction, and clonal reproduction, respectively; $d_{i j}^{t}=$ $\bar{t}_{i j} N_{j} / N_{i}^{\prime}, d_{i j}^{f}=\bar{f}_{i j} N_{j} / N_{i}^{\prime}$, and $d_{i j}^{c}=\bar{c}_{i j} N_{j} / N_{i}^{\prime} ; \mathbf{R}_{i j}$ is the diagonal matrix with entries $\rho_{i j k}$; and $\mathbf{I}$ is the $m \times m$ identity matrix (details are given in app. B, pt. A, which uses results from app. A, pt. A). Equation (2) shows that the association parameters $\rho_{i j k}$ (collected in matrix $\mathbf{R}_{i j}$ ) partition the effects on the change in mean phenotype from clonal reproduction into separate terms that are either phenotype dependent (as are transitions) or genotype dependent (as is sexual reproduction). The two summation terms in equation (2) reflect different contributions to the mean phenotype over one time step. The first summation is a weighted average of the stage-specific mean phenotypes (terms involving $\overline{\mathbf{z}}_{j}$ ) and genotypes (terms involving $\overline{\mathbf{g}}_{j}$ ) contributing to stage $i$. The phenotype-dependent terms include both transitions (which obviously involve the phenotype of the individual transitioning from stage $j$ to stage $i$ ) and the proportion of clonal reproduction that reflects phenotype, as determined by the association parameters in the matrix $\mathbf{R}_{i j}$. The genotypedependent terms include sexual reproduction (which under random mating only transmits the genotypes of the parents) and the proportion of clonal reproduction that reflects parental genotypes, measured by $\mathbf{I}-\mathbf{R}_{i j}$. The second summation includes terms with gradients of fitness components against mean phenotype and thus measures the contribution to the mean phenotype of selection from the previous time step.

We note that the derivation of the stage-structured form of Price's equation given in Barfield et al. (2011) carries over to the model considered here. Additionally, Lande's theorem for stage-structured populations, as derived in Barfield et al. (2011), also applies to the model with clonality. That is to say, in this more general model it is still the case (given assumptions laid out there) that $\Delta \overline{\mathbf{z}}=\left(\sum_{i, j} v_{i} w_{j} \mathbf{G}_{j} \nabla_{\overline{\mathbf{z}}_{j}} \bar{a}_{i j}\right) / \bar{\lambda}$, where $\Delta \overline{\mathbf{z}}$ is the asymptotic rate of phenotypic change of all stages assuming a steady state has been reached and, for the transition matrix with elements $\bar{a}_{i j}, \bar{\lambda}$ is its dominant eigenvalue and $\mathbf{w}=\left\{w_{i}\right\}$ and $\mathbf{v}=\left\{v_{i}\right\}$ are its right and left eigenvectors, normalized so that $\mathbf{v}^{\mathrm{T}} \mathbf{w}=1$. (Note that the definitions of some terms in the first paragraph of app. B of Barfield et al. [2011] differ from those presented herenotably, $\boldsymbol{\beta}_{z}, \mathbf{C}^{t}$, and $\mathbf{C}^{f}$ - but these different definitions have no effect on the subsequent derivation or final result.) Thus, these general results from evolutionary theory are robust to mixtures of clonal and sexual reproductive strategies.

\section{Simple Life-History Examples \\ Nonoverlapping Generations and Clonal Reproduction: Analytical Results}

To illustrate the implications of our general results (e.g., eqq. [1] and [2]), we now consider a simple life history with clonal reproduction but nonoverlapping generations, which can be represented by a single life-history stage (see fig. $1 A$ ). To simplify the analysis further, we consider a single quantitative trait $(z)$ rather than the multivariate trait considered above ( $\mathbf{z}$ ); thus, the gradients in the phenotype and genotype recursions are replaced by simple derivatives, and the additivegenetic covariance matrix $\mathbf{G}$ and phenotypic covariance matrix $\mathbf{P}$ are replaced by genotypic and phenotypic variances $G$ and $P$. There is only one type of parent-clonal offspring association for this life history, which we denote $\rho$. New individuals are formed via either sexual $(f)$ or clonal $(c)$ repro- 


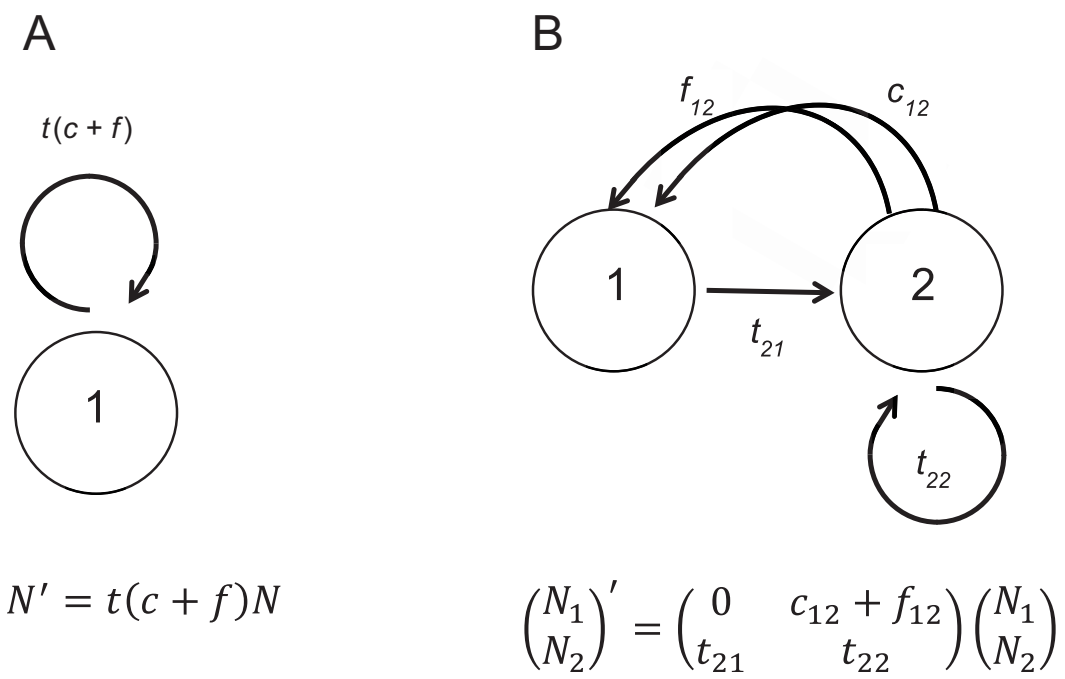

Figure 1: Life-cycle graph and population size recursions for simple life histories considered in equations (3) and (7). A, One-stage life history with clonal and sexual reproduction but no stage structure (nonoverlapping generations). $B$, Two-stage life history with clonal and sexual reproduction and adult survival.

duction. The probability of survival to adulthood (and reproduction) is given by the probability $t$. The number of clonal offspring in one generation produced per offspring in the previous generation is the product of the latter's probability of surviving to adulthood and the clonal reproductive rate $(t c)$, the number of sexual offspring is the product of the survival probability and the sexual reproduction rate $(t f)$, and thus the total number of individuals per individual in the previous generation is $t(c+f)$ (see fig. $1 A$ ). If these effective fecundities are denoted $t c(\bar{z})$ and $t f(\bar{z})$, where $\bar{z}$ is the mean phenotype of the single stage (and $\bar{g}$ its mean genotype), equations (1) and (2) lead to

$$
\begin{aligned}
& \bar{z}^{\prime}=\frac{N}{N^{\prime}}\{t c[\rho \bar{z}+(1-\rho) \bar{g}]+t f \bar{g} \\
&\left.+t c \frac{d \ln t c}{d \bar{z}}[\rho P+(1-\rho) G]+t f G \frac{d \ln t f}{d \bar{z}}\right\},
\end{aligned}
$$

$\bar{g}^{\prime}=\frac{N}{N^{\prime}}\left\{(t c+t f) \bar{g}+G\left[t c \frac{d \ln t c}{d \bar{z}}+t f \frac{d \ln t f}{d \bar{z}}\right]\right\}$.

The recursion for population size is $N^{\prime}=t(c+f) N$, so $N / N^{\prime}=1 /[t(c+f)]$. We assume that the two reproductive rates $c$ and $f$ are independent of phenotype (i.e., selection is via juvenile survival). Let $r_{c}=c /(c+f)$ be the relative amount of clonal reproduction (the fraction of offspring that are clonal); the relative amount of sexual reproduction is then $1-r_{c}=f /(c+f)$. Given no selection on the trait, all derivatives in equations (3) are 0 , resulting in

$$
\begin{aligned}
& \bar{z}^{\prime}=r_{c} \rho(\bar{z}-\bar{g})+\bar{g}, \\
& \bar{g}^{\prime}=\bar{g} .
\end{aligned}
$$

From equations (4), it can be shown that, assuming the mean phenotype and mean genotype initially differ, with no selection and assuming $r_{c} \rho<1$, the difference between the mean phenotype and mean genotype decreases each generation, approaching a steady state at which the mean phenotype equals the mean genotype. Thus, in the absence of selection the steady-state mean genotype and phenotype for this simple life history do not depend on the association between the environmental (random) phenotypic component of each clonal offspring and that of its parent. The trajectories depend only on the initial values of these means and on the product of the relative amount of clonal reproduction and the parent-clonal offspring association $\left(r_{c} \rho\right)$, with a higher value of this quantity (more clonal reproduction, a closer match between the parent and clonal offspring environmental component of phenotype, or both) giving a slower approach to the steady state.

We are of course most interested in nonequilibrium cases where there is movement away from this equilibrium due to environmental change and selection. Let us assume that selection acts only on survival to adulthood, with $t=$ $t_{\max } \exp \left[-(\bar{z}-\theta)^{2} /\left(2 \omega^{2}\right)\right]$, where $t_{\max }$ gives the maximum survival rate, achieved if the mean phenotype is at its optimalvalue $\theta$, and $\omega$ is the width of the survival function. Then $t c=t_{\max } c \exp \left[-(\bar{z}-\theta)^{2} /\left(2 \omega^{2}\right)\right], d \ln t c(\bar{z}) / d \bar{z}=(\theta-\bar{z}) / \omega^{2}$, $t f=t_{\max } f \exp \left[-(\bar{z}-\theta)^{2} /\left(2 \omega^{2}\right)\right]$, and $d \ln t f(\bar{z}) / d \bar{z}=(\theta-$ $\bar{z}) / \omega^{2}$. Substituting these into equations (3) gives 
$\bar{z}^{\prime}=r_{c} \rho \bar{z}+\left(1-r_{c} \rho\right) \bar{g}+\left\{r_{c} \rho P+\left(1-r_{c} \rho\right) G\right\} \frac{\theta-\bar{z}}{\omega^{2}}$,

$\bar{g}^{\prime}=\bar{g}+G \frac{\theta-\bar{z}}{\omega^{2}}$

from which the rates of change of mean phenotype and genotype can be found, giving

$\Delta \bar{z}=\left(1-r_{c} \rho\right)(\bar{g}-\bar{z})+\left\{r_{c} \rho P+\left(1-r_{c} \rho\right) G\right\} \frac{\theta-\bar{z}}{\omega^{2}}$,

$\Delta \bar{g}=G \frac{\theta-\bar{z}}{\omega^{2}}$.

Below, we give the results of simulations using an individualbased model for a population with a life history corresponding to this analytical model, subject to a change in its optimal phenotype. One such change is a sudden step increase in the optimal phenotype, from 0 to a positive value $\theta$, after which the above equations provide an analytical approximation of the population's dynamics. Thus, if initially $\bar{z}=\bar{g}=0$, after the first generation the change in genotype is $G \theta / \omega^{2}$, and the change in phenotype is $\left\{r_{c} \rho P+\left(1-r_{c} \rho\right) G\right\} \theta / \omega^{2}$. The change in phenotype will thus be the same as the change in genotype if $r_{c} \rho=0$ and greater than the change in genotype otherwise (assuming $P>G$, as expected). If either the relative amount of clonal reproduction or the parent-clonal offspring association is 0 (so that $r_{c} \rho=0$ ), the genotype and phenotype will evolve at the same rate and, since they start at the same value, will remain equal. If $r_{c} \rho>0$, the mean phenotype will exceed the mean genotype one generation after the change in optimal phenotype; in each subsequent generation, the change in mean phenotype due to selection (the second term in eq. [6a]) will be larger than the change in mean genotype. However, if instead of starting with equal mean phenotype and genotype we start with $\bar{z}>\bar{g}$, the first term in equation (6a) is negative (unless $r_{c} \rho=1$ ), limiting divergence between phenotype and genotype. Soon after the change in optimal phenotype, the second term in equation (6a) will be greater than the first, but over time the second term will decrease as selection brings the mean phenotype closer to the optimum. As the second term drops in value, eventually the first term will outweigh the difference between the change in genotype and phenotype due to selection, and the difference between mean genotype and phenotype will decrease.

Thus, the existence of clonal reproduction with an association between the parent and offspring environmental component causes the phenotype to initially evolve more quickly than the genotype and more quickly than without clonal reproduction. Additionally, the genotype will evolve slower after the first generation, since its rate of evolution is lowered with a higher mean phenotype. Eventually, evolution of both phenotype and genotype will slow, and they both will ap- proach the optimal phenotype. By taking the difference between the recursions given above for mean phenotype and genotype in equations (5), we obtain $(\bar{z}-\bar{g})^{\prime}=r_{c} \rho(\bar{z}-\bar{g})+$ $r_{c} \rho(P-G)(\theta-\bar{z}) / \omega^{2}$, which indicates that if the mean phenotype is greater than the mean genotype (as will be the case the first generation following a step change), the genotype cannot become greater than the phenotype unless the mean phenotype overshoots the optimum.

Examples of results of the analytical model given by equations (5) are shown in figure 2. Note that with clonal reproduction and clonal offspring association, the phenotype initially evolves faster and the genotype slower than without clonality (fig. $2 \mathrm{~A}$ ); in the model, note that with greater clonality, selection on the phenotype results in increased response to selection (eq. [6a]), but this in turn slows the response at the genotypic level (eq. [6b]). As the difference between the phenotype and genotype increases, phenotypic evolution slows, and both the phenotype and
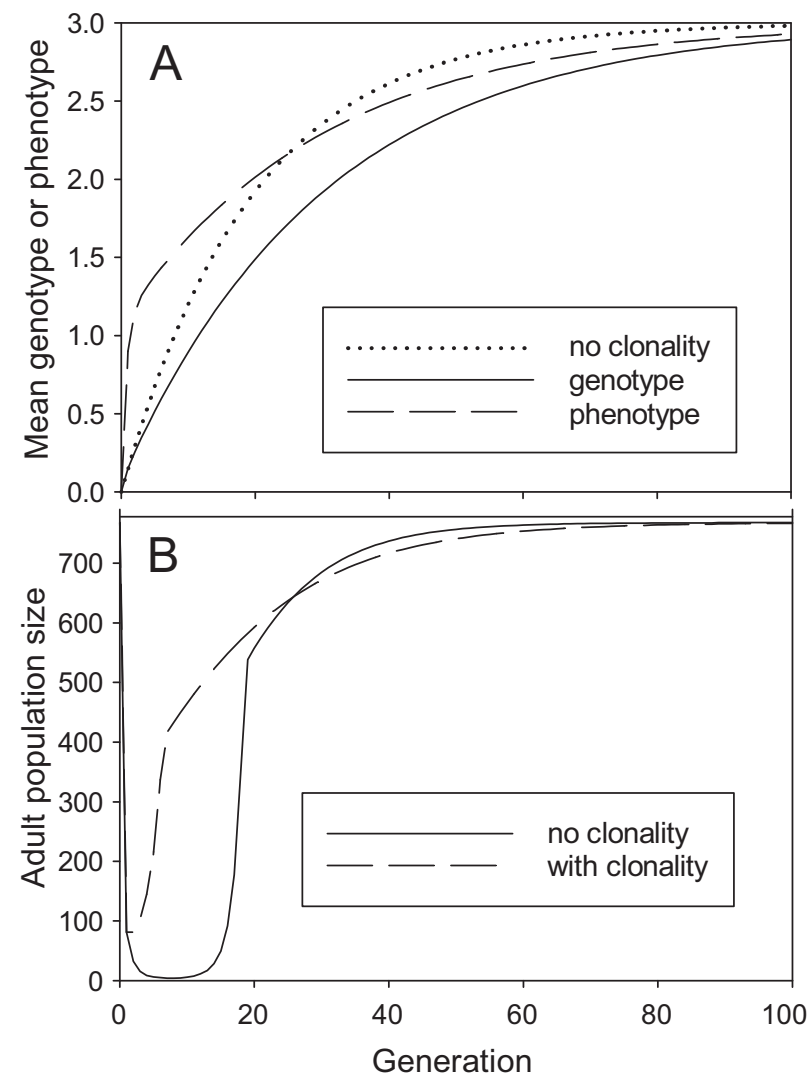

Figure 2: Example of results of the analytical model with nonoverlapping generations (eqq. [5]). $A$, Mean genotype and phenotype with $r_{c} \rho=0$ (dotted line) and $r_{c} \rho=0.5$ (solid line for genotype, dashed line for phenotype). $B$, Adult population size with $r_{c} \rho=0$ (solid line) and $r_{c} \rho=0.5$ (dashed line), with initial population of 768 and a maximum of 256 reproducing adults. Other parameters are $P=1.1, G=0.1, \theta=3, f=4, \omega^{2}=2, t_{\max }=0.75$, and, initially, $\bar{g}=\bar{z}=0$. 
the genotype eventually lag behind their values without clonality (the genetic variance for fig. 2 is low, limiting the genotypic rate of increase; after the first few generations, the phenotypic increase slows because part of the phenotype is due to the genotype of the parents and also because selection is weaker as $\bar{z}$ gets closer to $\theta$ ). Although the approach to the optimum is slower with clonality, population survival would be expected to be greater because survival is predominantly determined by the level to which the population size falls soon after the phenotypic optimum changes, and the faster increase in phenotype at that time under clonality increases fitness more rapidly and limits the drop in population size (fig. 2B).

\section{Simple Life-History Including Stage Structure: Analytical Results}

To explore the effects of overlapping generations and stage structure in addition to clonal reproduction, we next consider an extension of this simple model to an organism with two life-history stages, juveniles (stage 1) and adults (stage 2; see fig. $1 B$ ). Juveniles are formed via either sexual reproduction (produced at rate $\bar{f}_{12}$ per reproducing adult per time step) or clonal reproduction (produced at rate $\bar{c}_{12}$ ). Individuals move from the juvenile class to the adult class with transition probability $\bar{t}_{21}$ (but do not reproduce until the next time step), and adults survive from one time unit to the next with probability $\bar{t}_{22}$. The change in mean phenotype and mean genotype for juveniles and adults is then given by

$$
\begin{aligned}
\bar{z}_{1}^{\prime}= & \frac{N_{2}}{N_{1}^{\prime}}\left\{\bar{c}_{12}\left[\rho \bar{z}_{2}+(1-\rho) \bar{g}_{2}\right]+\bar{f}_{12} \bar{g}_{2}+\bar{c}_{12} \frac{d \ln \bar{c}_{12}}{d \bar{z}_{2}}\right. \\
& \left.\times\left[\rho P_{2}+(1-\rho) G_{2}\right]+\bar{f}_{12} G_{2} \frac{d \ln \bar{f}_{12}}{d \bar{z}_{2}}\right\}, \\
\bar{z}_{2}^{\prime}= & \frac{N_{1}}{N_{2}^{\prime}}\left\{\bar{t}_{21} \bar{z}_{1}+\bar{t}_{21} P_{1} \frac{d \ln \bar{t}_{21}}{d \bar{z}_{1}}\right\} \\
& +\frac{N_{2}}{N_{2}^{\prime}}\left\{\bar{t}_{22} \bar{z}_{2}+\bar{t}_{22} P_{2} \frac{d \ln \bar{t}_{22}}{d \bar{z}_{2}}\right\}, \\
\bar{g}_{1}^{\prime}= & \frac{N_{2}}{N_{1}^{\prime}}\left\{\left(\bar{c}_{12}+\bar{f}_{12}\right) \bar{g}_{2}+G_{2}\left[\bar{c}_{12} \frac{d \ln \bar{c}_{12}}{d \bar{z}_{2}}+\bar{f}_{12} \frac{\left.d \ln \bar{f}_{12}\right]}{d \bar{z}_{2}}\right]\right\}, \\
\bar{g}_{2}^{\prime}= & \frac{N_{1}}{N_{2}^{\prime}}\left\{\bar{t}_{21} \bar{g}_{1}+G_{1} \bar{t}_{21} \frac{d \ln \bar{t}_{21}}{\left.d \bar{z}_{1}\right\}}\right. \\
& +\frac{N_{2}}{N_{2}^{\prime}}\left\{\bar{t}_{22} \bar{g}_{2}+G_{2} \bar{t}_{22} \frac{d \ln \bar{t}_{22}}{\left.d \bar{z}_{2}\right\} .}\right.
\end{aligned}
$$

The stage-specific population size recursions are $N_{1}^{\prime}=$ $\left(\bar{c}_{12}+\bar{f}_{12}\right) N_{2}$ and $N_{2}^{\prime}=\bar{t}_{21} N_{1}+\bar{t}_{22} N_{2}$. Dividing the latter by its left side gives $1=\bar{t}_{21} N_{1} / N_{2}^{\prime}+\bar{t}_{22} N_{2} / N_{2}^{\prime}$. Assuming the population is at the steady-state stage distribution, $N_{2} / N_{2}^{\prime}=1 / \lambda$, we can solve $1=\bar{t}_{21} N_{1} / N_{2}^{\prime}+\bar{t}_{22} N_{2} / N_{2}^{\prime}$ for $N_{1} / N_{2}^{\prime}=\left(1-\bar{t}_{22} / \lambda\right) / \bar{t}_{21}$, where $\lambda$ is the leading eigenvalue of the life-history transition matrix with entries $\bar{a}_{i j}$,

$$
\lambda=\frac{1}{2}\left(\bar{t}_{22}+\sqrt{4 \bar{t}_{21}\left(\bar{c}_{12}+\bar{f}_{12}\right)+\bar{t}_{22}^{2}}\right)
$$

(which is the population growth rate at the steady-state distribution across stages).

If we assume no selection on the trait, all derivatives in equations (7) are 0 , and we then have

$$
\begin{aligned}
\bar{z}_{1}^{\prime} & =r_{c}\left[\rho \bar{z}_{2}+(1-\rho) \bar{g}_{2}\right]+\left(1-r_{c}\right) \bar{g}_{2} \\
& =r_{c} \rho\left(\bar{z}_{2}-\bar{g}_{2}\right)+\bar{g}_{2}, \\
\bar{z}_{2}^{\prime} & =\bar{z}_{1}+\frac{\bar{t}_{22}}{\lambda}\left(\bar{z}_{2}-\bar{z}_{1}\right), \\
\bar{g}_{1}^{\prime} & =\bar{g}_{2}, \\
\bar{g}_{2}^{\prime} & =\bar{g}_{1}+\frac{\bar{t}_{22}}{\lambda}\left(\bar{g}_{2}-\bar{g}_{1}\right) .
\end{aligned}
$$

At steady state, $\bar{z}_{1}^{\prime}=\bar{z}_{1}, \bar{z}_{2}^{\prime}=\bar{z}_{2}, \bar{g}_{1}^{\prime}=\bar{g}_{1}$, and $\bar{g}_{2}^{\prime}=\bar{g}_{2}$. From equation (8b), we can show that $\bar{z}_{2}=\bar{z}_{1}$; using this, equation (8a) gives $\bar{z}_{2}=\bar{g}_{2}$. Equation (8c) gives $\bar{g}_{1}=\bar{g}_{2}$, as does equation ( $8 \mathrm{~d})$. Therefore, the values of all four means converge; numerical iteration of equations (8) show that this convergence occurs with damped oscillations with a twotime-step period (results not shown).

To explore how selection affects the model given in equations (7), we begin by assuming that selection acts only on survival of juveniles to adulthood (as before), so that $\bar{t}_{21}=t_{\max } \exp \left[-\left(\bar{z}_{1}-\theta\right)^{2} /\left(2 \omega^{2}\right)\right]$. Then $d \ln t_{21}\left(\bar{z}_{1}\right) / d \bar{z}_{1}=$ $\left(\theta-\bar{z}_{1}\right) / \omega^{2}$, and once again the stage-specific population size recursions are $N_{1}^{\prime}=\left(\bar{c}_{12}+\bar{f}_{12}\right) N_{2}$ (and thus $N_{2} / N_{1}^{\prime}=$ $\left.1 /\left(\bar{c}_{12}+\bar{t}_{12}\right)\right)$ and $N_{2}^{\prime}=\bar{t}_{21} N_{1}+\bar{t}_{22} N_{2}$. Substituting these into equations (7) leads to

$$
\begin{aligned}
\bar{z}_{1}^{\prime}= & r_{c} \rho\left(\bar{z}_{2}-\bar{g}_{2}\right)+\bar{g}_{2}, \\
\bar{z}_{2}^{\prime}= & \bar{z}_{2}+\frac{N_{1}}{\bar{t}_{21} N_{1}+\bar{t}_{22} N_{2}} \bar{t}_{21} \\
& \times\left[\left(\bar{z}_{1}-\bar{z}_{2}\right)+P_{1}\left(\frac{\theta-\bar{z}_{1}}{\omega^{2}}\right)\right], \\
\bar{g}_{1}^{\prime}= & \bar{g}_{2}, \\
\bar{g}_{2}^{\prime}= & \bar{g}_{2}+\frac{N_{1}}{\bar{t}_{21} N_{1}+\bar{t}_{22} N_{2}} \bar{t}_{21} \\
& \times\left[\left(\bar{g}_{1}-\bar{g}_{2}\right)+G_{1}\left(\frac{\theta-\bar{z}_{1}}{\omega^{2}}\right)\right],
\end{aligned}
$$


where $\bar{t}_{22}$ is assumed to be fixed. When the phenotypic trait is at the optimum for juveniles, $\bar{t}_{21}=t_{\max }$ and there is no selection, and we return to equations (8). The composite parameter $r_{c} \rho$ again determines the amount by which the phenotype (now the juvenile trait mean) differs from the genotype; if $r_{c} \rho=0$, then $\bar{z}_{1}^{\prime}=\bar{g}_{1}^{\prime}=\bar{g}_{2}$, and if $r_{c} \rho=1$, then $\bar{z}_{1}^{\prime}=\bar{z}_{2}$ and $\bar{g}_{1}^{\prime}=\bar{g}_{2}$. Substituting equation (9c) into equation (9d) at equilibrium gives $\bar{z}_{1}=\theta$, which after substitution into equation ( $9 \mathrm{~b}$ ) gives $\bar{z}_{1}=\bar{z}_{2}$. As long as $r_{c} \rho \neq$ 1, equation (9a) gives $\bar{z}_{i}=\bar{g}_{i}$ for each stage. Thus, unless $r_{c} \rho=1$, all four quantities approach $\theta$ at equilibrium (if $r_{c} \rho=1$, the phenotypes still approach $\theta$, but the genotypes can go to a different value). Given that all four means move to the same equilibrium value, the effect of clonal reproduction is thus to change only the shape of the deterministic trajectories on their way to equilibrium.

Examples for the analytical model given by equations (9) with $t_{\max }$ fixed are shown in figure $3 A$ and $3 B$ for three prob- abilities of adult survival $\left(\bar{t}_{22}=0.1,0.25\right.$, and 0.5$)$, with $r_{c} \rho=0.25$. Figure $3 A$ shows adult-stage mean genotypes and phenotypes $\left(\bar{g}_{2}\right.$ and $\left.\bar{z}_{2}\right)$, and figure $3 B$ shows adult population size ( $N_{2}$ after ceiling density dependence). The initial value of $\bar{t}_{21}$ (when $\bar{z}_{1}$ is 0 and its optimum, $\theta$, is 3 ) is 0.07905 ; selection moves the value of $\bar{t}_{21}$ to $0.75\left(t_{\max }\right)$ when $\bar{z}_{1}$ approaches its equilibrium value of 3 . Increased adult survival leads to slower evolution; we observe a slower approach for $\bar{g}_{2}$ and $\bar{z}_{2}$ to their equilibrium values with increasing $\bar{t}_{22}$ (fig. 3A). The effect of adult survival on these deterministic results is via the term $N_{1} / N_{2}^{\prime}$ in equations (9b) and (9d). When we increase $\bar{t}_{22}$, we are increasing $N_{2}^{\prime}$ and decreasing $N_{1} / N_{2}^{\prime}$, weakening the effect of selection and also decreasing the rate at which $\bar{z}_{2}$ and $\bar{z}_{1}$ and at which $\bar{g}_{2}$ and $\bar{g}_{1}$, respectively, approach one another. However, increased adult survival dramatically decreases both the severity and the duration of the population size decline caused by the initial strong selection on $\bar{t}_{21}$ (when $\bar{z}_{1}=0$ and $\theta=3$; see

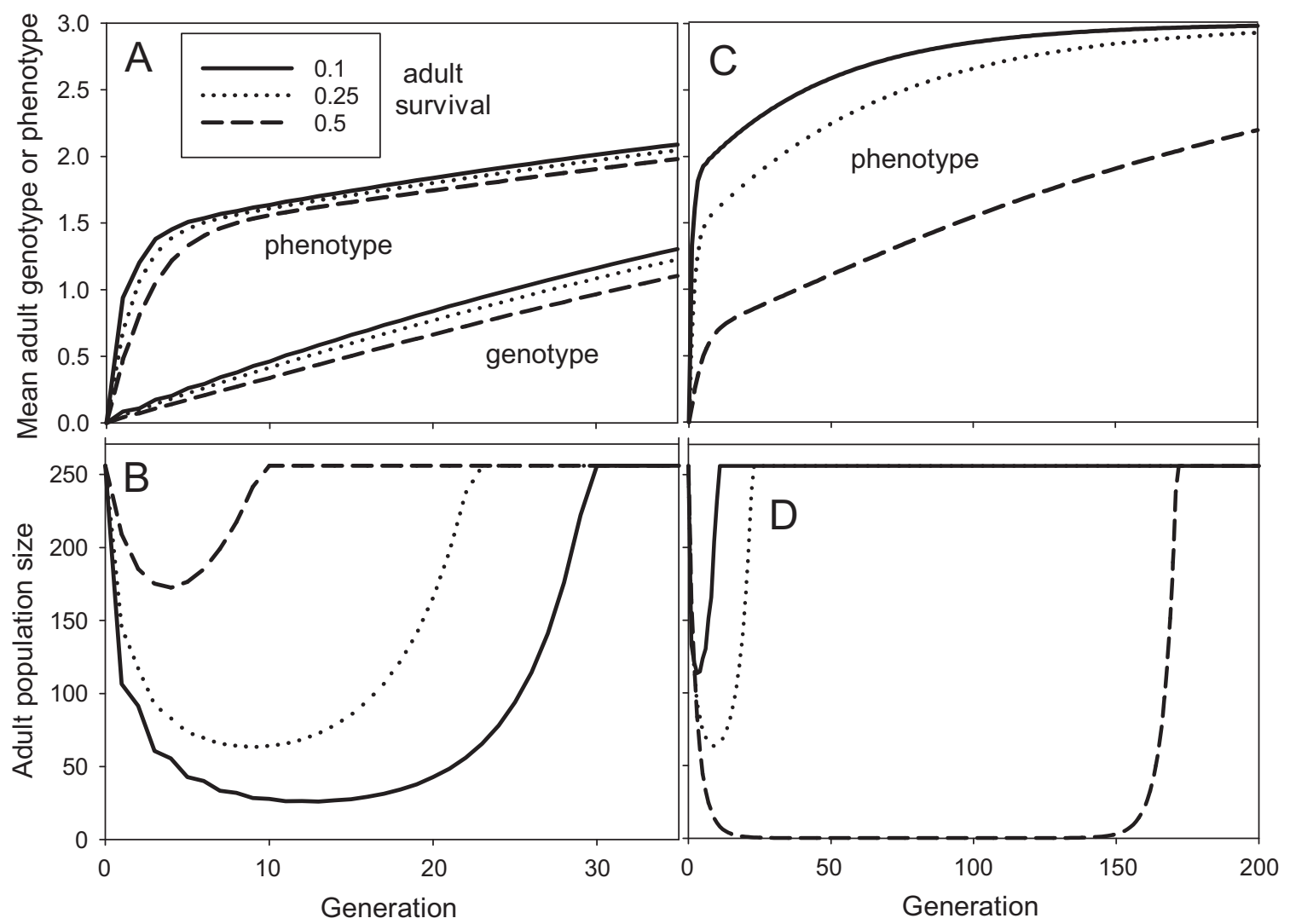

Figure 3: Example of results of the two-stage analytical model with overlapping generations (eqq. [9]). $A, B$, Adult-stage mean genotypes and phenotypes $(A)$ and adult population size (after ceiling density dependence; $B$ ) with different values of adult survival. Parameters are $\bar{c}_{12}=\bar{f}_{12}=2$ (giving $r_{c}=0.5$ ), $P_{2}=1.1, G_{2}=0.1, \rho=0.5, \theta=3, \omega^{2}=2$, and $t_{\max }=0.75$. Initial values are $N_{1}=1,024, N_{2}=256$, and $\bar{z}_{1}=\bar{z}_{2}=\bar{g}_{1}=$ $\bar{g}_{2}=0 . N_{2}$ is not allowed to exceed 256. C, D, Adult stage mean phenotypes (C) and adult population size (after ceiling density dependence; $D)$ for the two-stage analytical model with different values of adult survival, with $t_{\max }$ adjusted to keep the initial decline at the stable stage distribution $(\lambda)$ the same: $t_{\max }=0.9994,0.75$, and 0.334 for $\bar{t}_{22}=0.1,0.25$, and 0.5 , respectively. All other parameters and initial values are the same as in $A$ and $B$. 
fig. $3 B$ ). This demographic effect outweighs the evolutionary effect of increased adult survivorship, allowing a higher minimum population size and a decreased likelihood of extinction.

If the initial rate of population decline were kept constant (by decreasing either fecundity or the maximum juvenile survival as adult survival increases), then higher adult survival leads to a lower minimum population size and therefore a higher risk of extinction. An example where maximum juvenile survival $\left(t_{\max }\right)$ is decreased as adult survival $\left(\bar{t}_{22}\right)$ is increased to keep the initial decline at the stable stage distribution $(\lambda)$ constant is shown in figure $3 C$ and $3 D$. In this case, increased adult survival again slows evolution, as in figure $3 A$, but keeping the initial rate of decline fixed also eliminates the demographic advantage of adult survival (compare fig. $3 B$ with $3 D$ ). The population size is now reduced more severely with increasing adult survival, increasing extinction risk prior to adaptation to the new phenotypic optimum.

Comparing adult population size for the same adult survival rate but with or without clonality $\left(r_{c} \rho>0\right.$ vs. $\left.r_{c} \rho=0\right)$ shows that nonzero values of $r_{c} \rho$ also cause less severe drops in population size and a more rapid population recovery for the two-stage model, as in the one-stage model with no adult survival (results not shown). Hence, increased phenotypic association between a parent and its clonal offspring can facilitate population persistence in an abruptly changed environment.

\section{Exploration of Evolutionary Rescue and Lag under a Changing Optimal Phenotype}

\section{Individual-Based Simulations}

The models discussed above neglect important aspects of stochasticity in both evolution (e.g., mutation, drift) and demography (demographic stochasticity leading to extinction events). To consider the consequences of such stochasticity and to further explore the implications of the lifehistory strategies considered above for evolutionary rescue and lag, we carried out simulations based on an individualbased approach used by Burger and Lynch (1995; see also Holt et al. 2005). We examine several distinct scenarios of environmental change. The details of the individual-based simulations are laid out in appendix A, part B, so we provide a schematic overview here. The simulations tracked individuals (hermaphrodites) and their genotypes and phenotypes in a single population with density-dependent regulation (a population size "ceiling" on the number of mating adults, below which there are density-independent dynamics). Reproduction could be partially sexual and partially clonal, with gametic and somatic mutations. Selection acts on a single polygenic trait (survival from juvenile to adult, which was a Gaussian function of the difference between an individual's trait value and an optimal phenotype) governed by $n$ diploid unlinked loci (the examples shown all assume $n=10$ ); allelic effects were assumed to be additive both within and among the loci, so that no dominance or epistatic effects were considered for the phenotypic trait. (We note that our use of a Gaussian fitness surface, described in app. A, pt. B, creates epistasis for fitness, and we return to this point in "Discussion.") The nonadditive genetic plus environmental deviation (e) for sexual offspring was assumed to be normally distributed with mean 0 and variance 1 . For clonal reproduction, values of $e$ for an offspring could reflect those of its parent. The "cost" of a clonal offspring relative to a sexual offspring was given by parameter $\delta$; if clonal reproduction matched sexual reproduction, $\delta=1$, while if clonal offspring were more costly, $\delta>1$ (and thus for the same effort clonal reproduction led to fewer offspring than sexual reproduction; $r_{c}$ is the fraction of reproductive effort that is clonal). Each population was allowed to reach equilibrium before the optimal phenotype changed. Genotypic and phenotypic distributions over each population arise from the simulation of individuals undergoing mutation, segregation, selection, and drift and so are not necessarily Gaussian.

Two types of environmental shifts were examined: a onestep change in the optimal phenotype, after which the environment remained constant, and a linear, continuous change. For both types of change, we considered the role played by clonal reproduction in determining population persistence under a changing phenotypic optimum by varying the fraction of reproductive effort that was clonal, $r_{c}$, and the clonal offspring association parameter $\rho$. Both populations with nonoverlapping generations (mirroring the model given in eqq. [3] above) and those with a two-stage structure with adult survival (similar to eqq. [7] above) were examined.

\section{One-Step Change in Optimal Phenotype}

To assess the effect of clonal reproduction on the probability of population persistence after a single step change in the optimal phenotype, 400 simulation runs were performed for each parameter set so as to estimate the probability of population survival over 1,000 time steps. When sexual and clonal offspring were equally costly $(\delta=1)$, increasing the relative amount of clonal reproduction $\left(r_{c}\right)$ increased the probability of persistence (fig. $4 A$ ). However, if clonal offspring were more costly than sexual offspring, we observed a switch in this pattern. In figure $5 A, \delta=1.5$ (clonal offspring require $50 \%$ more effort); here, increasing $r_{c}$ decreased the probability of persistence after a step change in the optimal phenotype. Under this scenario $(\delta>1)$, increasing the relative amount of clonal reproduction led to fewer total offspring, so there was an overall decrease in fitness as $r_{c}$ increased. For a given amount of clonal reproduc- 

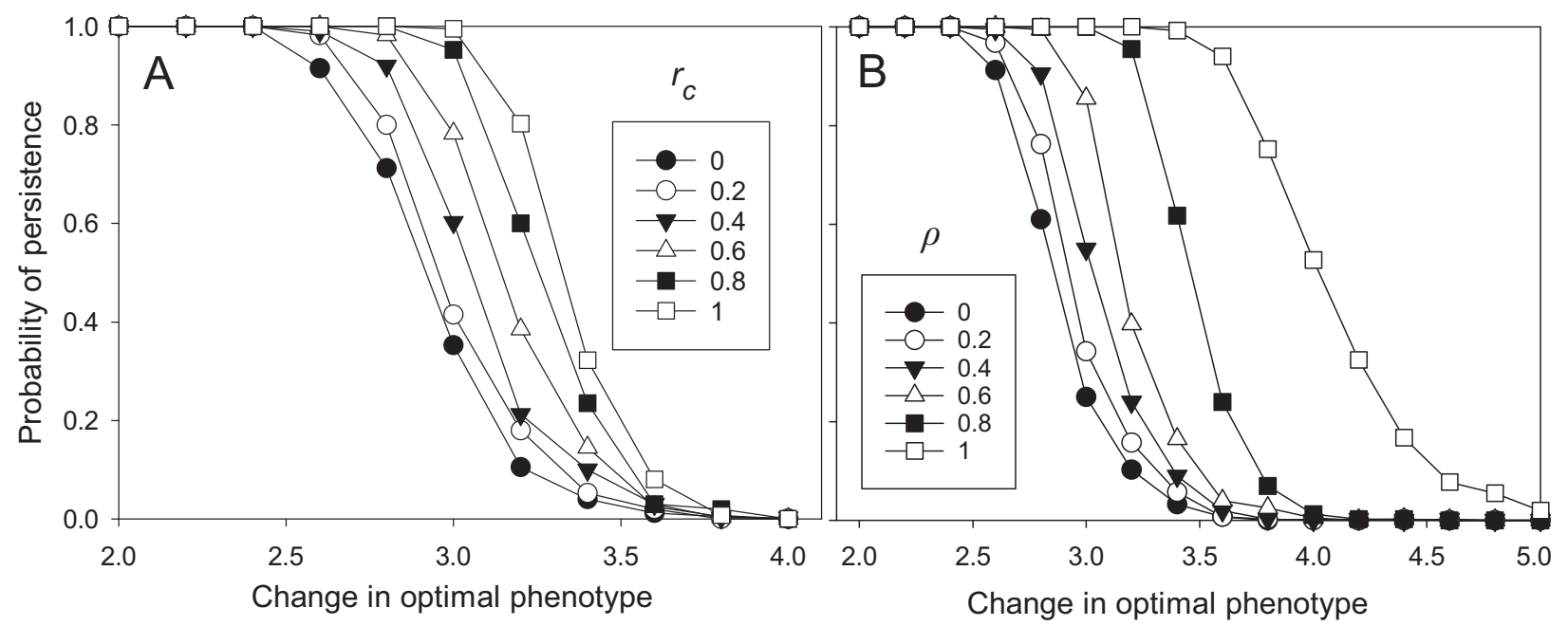

Figure 4: Probability of population persistence after a one-step change in optimal phenotype with equal effort for sexual and clonal reproduction $(\delta=1)$ and nonoverlapping generations as a function of the magnitude of the change $(\theta)$, using the individual-based model with parameters $K=256, f=4, \omega^{2}=1, n=10, \mu_{g}=0.001, \mu_{s}=0.00001, \alpha^{2}=0.05$, and $G=1,000 . A$, Effect of varying the relative amounts of sexual and clonal reproductive effort $\left(r_{c}\right.$ is the clonal fraction) with association parameter $\rho=0.5$. B, Effect of varying $\rho$ with $r_{c}=$ 0.5 .

tion (and with equally costly offspring), there was an increase in the probability of population persistence after a step change in the optimal phenotype as the association parameter between the parent and clonal offspring environmental component $(\rho)$ was increased (fig. $4 B$ ). This result matches our expectation based on the analytical model (eqq. [3] above). In all cases, however, a sufficiently large change in the optimal phenotype (corresponding to a suffi- ciently severe environmental change) made extinction inevitable.

For these results, reproduction was assumed to be completely sexual during the initialization period prior to the environmental change, which ensured that all populations had the same expected genetic variance at the time of the environmental change. Increasing clonality would be expected to lead to lower genetic variation (assuming the so-
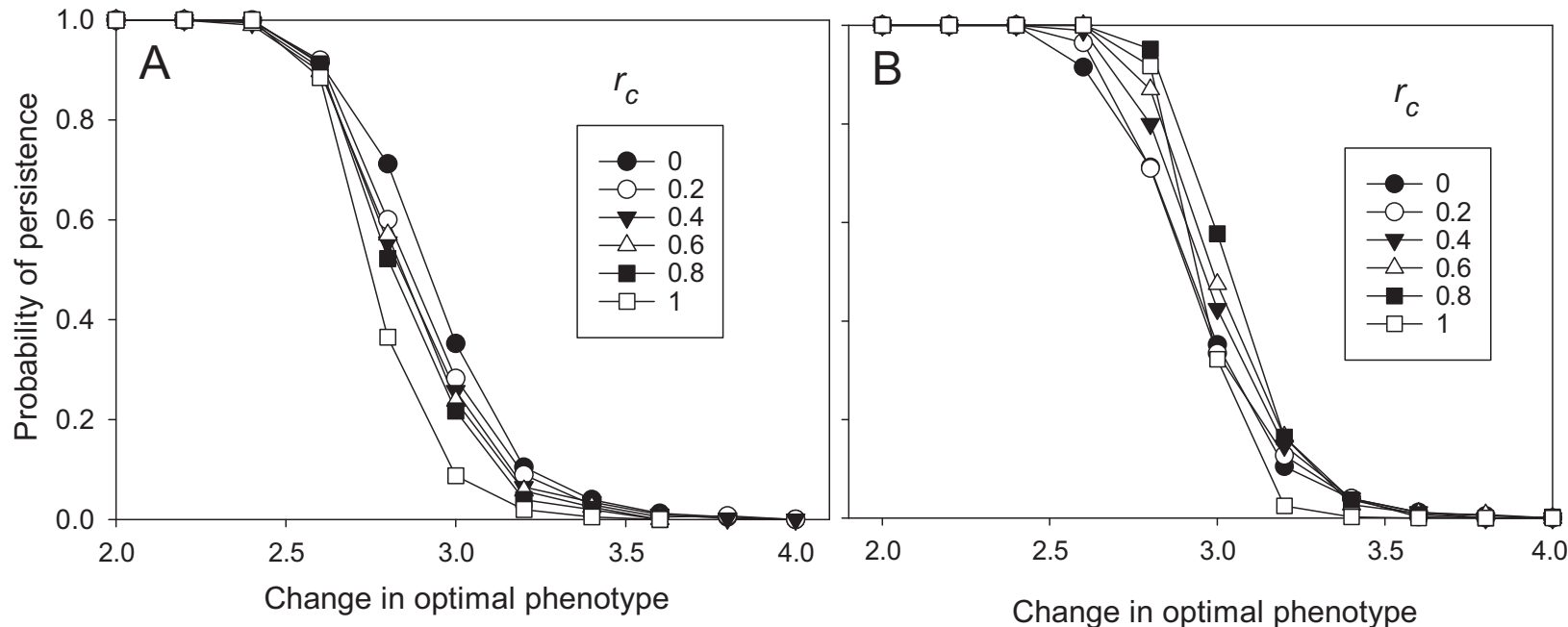

Figure 5: Probability of population persistence after a one-step change in optimal phenotype and nonoverlapping generations as a function of the magnitude of the change $(\theta)$ for different values of $r_{c}$, using the individual-based model with parameters as in figure 4 . In $A$, clonal reproduction requires $50 \%$ more effort $(\delta=1.5)$. In $B$, clonal and sexual effort are equal $(\delta=1)$, but the population has the value of $r_{c}$ given in the key from the start of the simulation. In both cases, $\rho=0.5$. 
matic mutation rate is lower than the gametic mutation rate), so some of the advantage of clonality could be lost if the initial population is partially or entirely clonal. An example for which the population had the same clonal fraction $\left(r_{c}\right)$ both before and after the step change in the optimum is shown in figure $5 B$. Compared with the equivalent case in which the population prior to the step change was fully sexual (shown in fig. $4 A$ ), the beneficial effect of increasing the relative proportion of clonal reproduction was decreased (the lines are shifted to the left), indicating that some of the benefit of clonal reproduction seen for a step change was lost when the genetic variance at the time of environmental change is reduced (due to the lower somatic mutation rate). In fact, with sufficiently large changes in the optimum phenotype, increased clonality hampers persistence when it also characterized the ancestral population (note that some of the curves cross in fig. 5B).

The beneficial effects of clonality can be understood as follows. After an abrupt environmental change, population persistence depends strongly on the standing heritable variation for the trait present just prior to the optimum shift. For individuals with phenotypes near or over the new phenotypic optimum, clonal offspring inherit a complete genotypic match and suffer from no segregational or recombination load, so that increased clonal reproduction enhances population persistence (as long as the phenotypic shift is not too great). Moreover, increased parent-clonal offspring association (increased $\rho$ ) causes an increased match in the nonadditive genetic plus environmental component as well, increasing the phenotypic match between favored parents and their clonal offspring. When standing variation exists that potentially allows a population to persist after a sudden shift in its optimal phenotype, increased genotypic matching (via clonal instead of sexual reproduction) and increased phenotypic matching (via increased parent-clonal offspring association) both increase the persistence of offspring of those beneficial variants in the next and subsequent generations, facilitating evolutionary rescue.

\section{Linear Change in Optimal Phenotype}

The results for a linear, continuous change in the optimal phenotype contrast starkly with those observed for a singlestep change. Even with no extra cost for clonal offspring $(\delta=1)$, increased clonal reproduction decreased population persistence for a given rate of change of the optimal phenotype and caused an increase in evolutionary lag, as measured by the realized difference between the average and optimal phenotypes in persisting populations (fig. 6A). In contrast to a step change, a successful long-term evolutionary response to continued change in the optimal phenotype requires de novo variation. In the simulation model considered here, there are three sources of novel variation: (1) recombination due to sexual reproduction (recall that there is no segregation effect here due to the assumption of additive allelic effects), (2) new beneficial mutations arising either via sexual reproduction (meiotic mutations arising during gamete formation, $\mu_{g}$ ) or via incorporation of somatic mutations in clonal offspring $\left(\mu_{s}\right)$, and (3) random variation due to the nonadditive and environmental component of the trait $(e)$.

Per-locus meiotic mutation rates have been estimated to be approximately one to two orders of magnitude greater

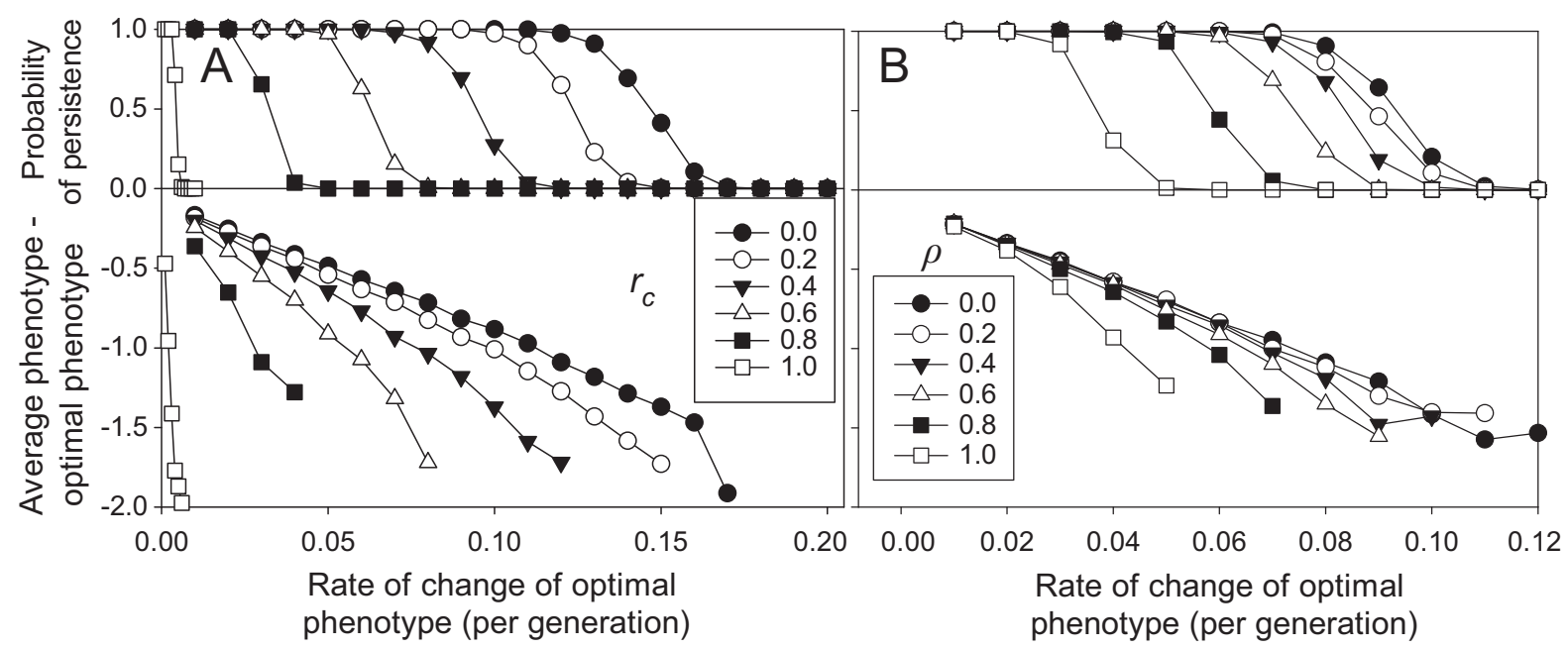

Figure 6: Probability of population persistence and evolutionary lag (the difference in average phenotype and optimal phenotype averaged over the last 200 generations for the populations that survived until the end of the simulation) after a linear change in optimal phenotype with equal effort for sexual and clonal reproduction $(\delta=1)$ and nonoverlapping generations as a function of the rate of change, using the individual-based model with parameters as in figure 4. A, Effect of varying $r_{c}$ with $\rho=0.5$. B. Effect of varying $\rho$ with $r_{c}=0.5$. 
than per-locus mitotic mutation rates (Magni and Von Borstel 1962; Lindgren 1975; Rattray et al. 2015; see the discussion in Orive [2001] and references therein). For figure 6, the gametic (meiotic) mutation rate was 100 times the somatic (mitotic) mutation rate. To determine whether the pattern in figure $6 \mathrm{~A}$ was due to this difference in de novo mutation rates, we varied $r_{c}$ with the mutation rates equalized $\left(\mu_{g}=\mu_{s}\right)$ at the previous $\mu_{g}$ value. The increased overall beneficial mutation rate increased the probability of persistence (note the change in scale for the abscissa), but persistence still decreased with increasing clonal reproduction (fig. $7 A$, open symbols). Making clonal reproduction more costly, of course, made increased clonal reproduction even more disadvantageous for persistence (fig. 7A, filled symbols).

Increasing the parent-clonal offspring association, which decreases the amount of novel variation introduced via $e$, reduced the probability of population persistence and increased evolutionary lag (with $\mu_{g}=100 \mu_{s}$; fig. 6B); this again is the opposite of the results seen for a step change. We also examined the case in which clonal offspring and sexual offspring were equalized as much as possible by making them equally costly $(\delta=1)$, assuming no parent-clonal offspring association $(\rho=0)$ and setting both types of mutation to have equal rates $\left(\mu_{g}=\mu_{s}\right)$. In this scenario, sexual and clonal offspring are equivalent for two of the three sources of de novo variation (mutation and $e$ ). There was still a drop in the probability of population persistence with an increasing proportion of clonal reproduction (fig. $7 B$ ). However, this drop was large only for a high proportion of clonal reproduction $\left(r_{c} \geq 0.6\right)$; for $r_{c} \leq 0.4$, there was little effect of clonal reproduction, indicating that relatively small amounts of recombination via sexual reproduction suffice to offset the loss of novel variation due to clonal reproduction. Note that the abscissa scale for figure 7 starts at 0.1 in order to show differences in this region more clearly; for $r_{c}=0$ (open hexagons) in figure $7 \mathrm{~B}$, populations persist for a rate of change of up to only 0.03 (not shown). Increasing sexual reproduction from $0 \%$ to $20 \%$ (decreasing $r_{c}$ from 1.0 to 0.8 ) therefore has a very large effect on the maximal rate of change that allows persistence; any additional advantage of more sexual reproduction decreases rapidly thereafter. (Note that evolutionary lag in figs. 6 and 7 was averaged only over those populations that survived until the end of the simulation. Therefore, where the probability of persistence was very small, evolutionary lag values were averaged over few populations, accounting for the sudden changes in some of those final values.)

Finally, as noted above, for the simulations shown in figures 6 and 7 reproduction was completely sexual during the period prior to the initiation of the linear environmental change, which ensured that all populations have the same expected genetic variance initially. Simulations were also run with the initial population having the same relative amounts of sexual and clonal reproduction (same $r_{c}$ ) before and after the beginning of the environmental change. In contrast to the results observed for a step change in environment (fig. 5B), the results for a linear, continuous change with an initially partially clonal population were very similar to those for the initially completely sexual population simulations (results not shown). This makes
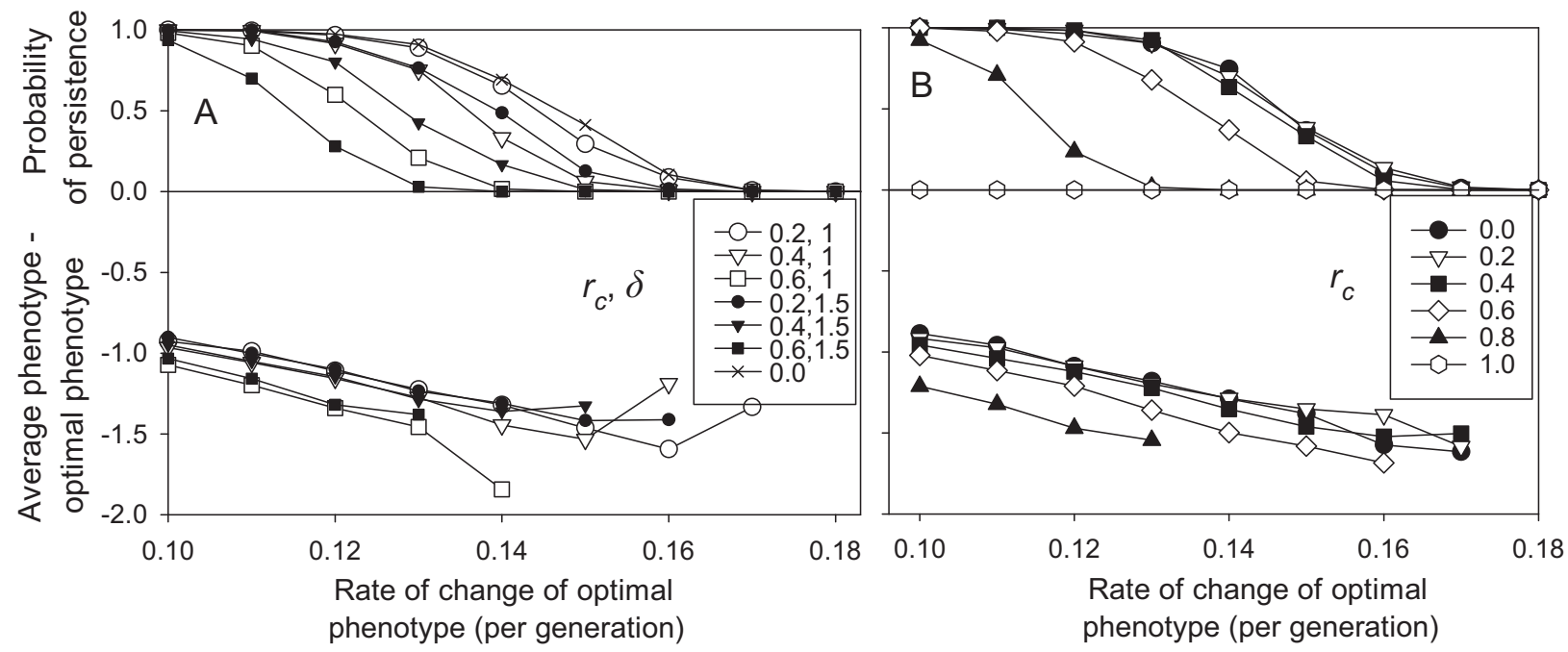

Figure 7: Probability of population persistence and evolutionary lag after a linear change in optimal phenotype with nonoverlapping generations as a function of the rate of change with somatic mutation rate equal to gametic mutation rate $\left(\mu_{g}=\mu_{s}=0.001\right)$, using the individual-based model with parameters other than $\mu_{s}$ as in figure 4. A, Effect of varying $r_{c}$ and $\delta$ with $\rho=0.5$. B, Effect of varying $r_{c}$ with $\delta=1$ and no association $(\rho=0)$. 
sense in light of the decreased importance of the standing heritable variation for the trait and the increased importance of de novo variation for surviving this type of environmental change.

\section{Including Stage Structure}

To consider the combined effects of stage structure and clonal reproduction, the individual-based simulations were modified to include a nonzero probability of adult survival ( $t_{22}$ in fig. $1 B$ ), as in the model given by equations (7), with selection only on survival from the juvenile to adult stages. Representative results are shown in figures 8 and 9. For a step change in the optimal phenotype (fig. 8), including adult survival and stage structure greatly increased the probability of population persistence for all values of the relative amount of clonal reproduction $\left(r_{c}\right)$ and the clonal offspring association parameter $(\rho)$; all of the curves in figure 8 are shifted to the right compared with the corresponding curves in figure 4 (note the difference in scale for the abscissa). Increasing adult survival (without a compensating change in either fecundity or juvenile survival; see below) increases the population growth rate $(\lambda)$ and leads to a demographic advantage, as discussed above for the analytical results including stage structure. In considering the effect of the relative amount of clonal reproduction, there was a greater effect on the probability of persistence for a given increase in $r_{c}$ with adult survival; the curves in figure $8 A$ are more widely spaced than those in figure $4 A$.

With a linear, continual change in the optimal phenotype, there again was a strong contrast in the effects of clonal reproduction compared with the step change. Now, the addition of adult survival decreased population persistence and increased the difference between the average phenotype and the optimal phenotype; the curves for figure 9 are shifted to the left compared with the corresponding curves in figure 6 , indicating that for these simulations, the same rate of change in the optimal phenotype led to a much lower probability of population persistence. Again in contrast to the one-step change results, we see that there is a smaller effect on the probability of persistence for a given increase in $r_{c}$ with adult survival; the curves in figure $9 A$ are more narrowly spaced than those in figure $6 A$ (note the change in scale).

Several distinct ecological and genetic effects that interact with clonal reproduction may help explain reduced population persistence with increased adult survival in continually changing environments. Increasing adult survival with no compensatory change in other life-history components leads to an increase in generation time ("meiotic generation time" for stage-structured populations; see Orive 1993 and Orive 1995 [appendix]). Under continual change, a longer generation time leads to more environmental change (a greater shift in the optimal phenotype) per generation and thus a greater amount of evolutionary lag. If populations are able to keep their numbers near carrying capacity (as commonly observed in the simulations with a linear change except when populations approached extinction; results not shown), having adults that survive into the next time step automatically reduces the number of juveniles (from both sexual and clonal reproduction) that can establish. This is because new adults and those surviving from the previous time step compete for the limited breeding slots near carrying

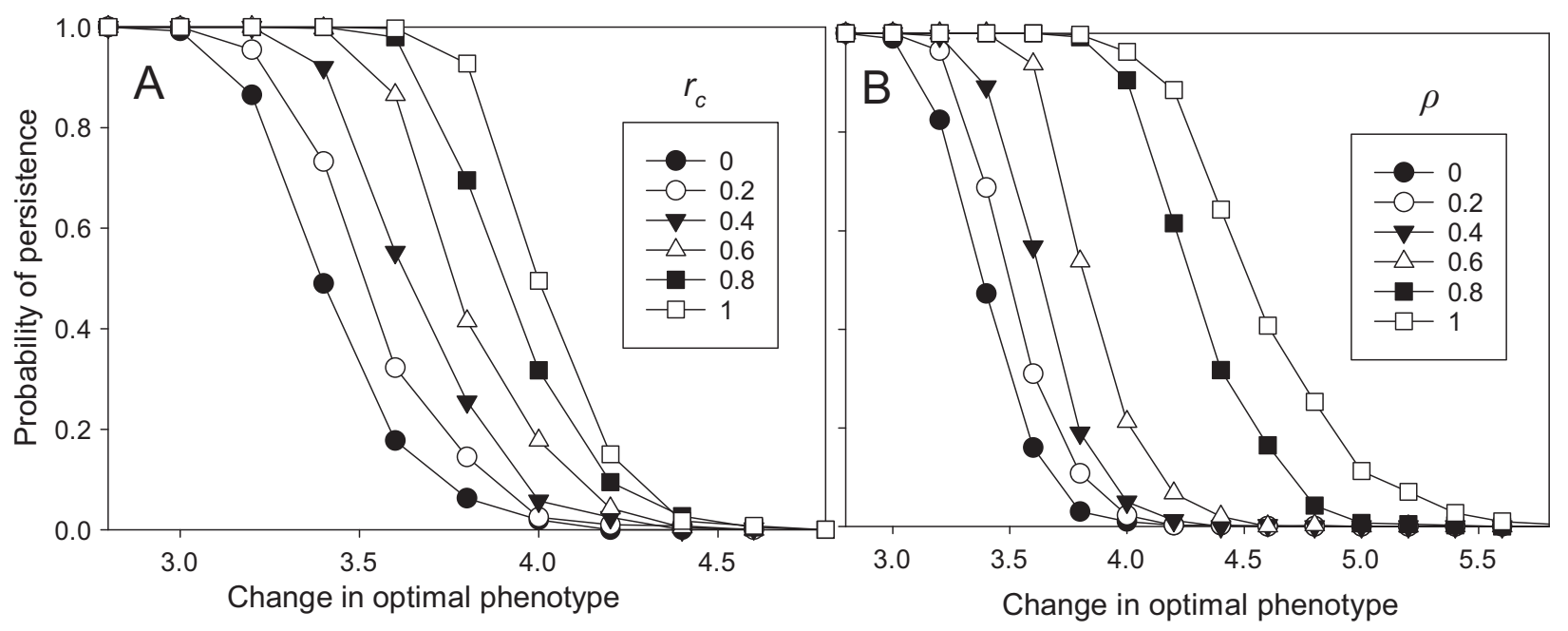

Figure 8: Probability of population persistence after a one-step change in optimal phenotype with equal effort for sexual and clonal reproduction $(\delta=1)$ and $50 \%$ adult survival as a function of the magnitude of the change $(\theta)$, using the individual-based model with parameters as in figure 4. A, Effect of varying $r_{c}$ with $\rho=0.5$. B, Effect of varying $\rho$ with $r_{c}=0.5$. 


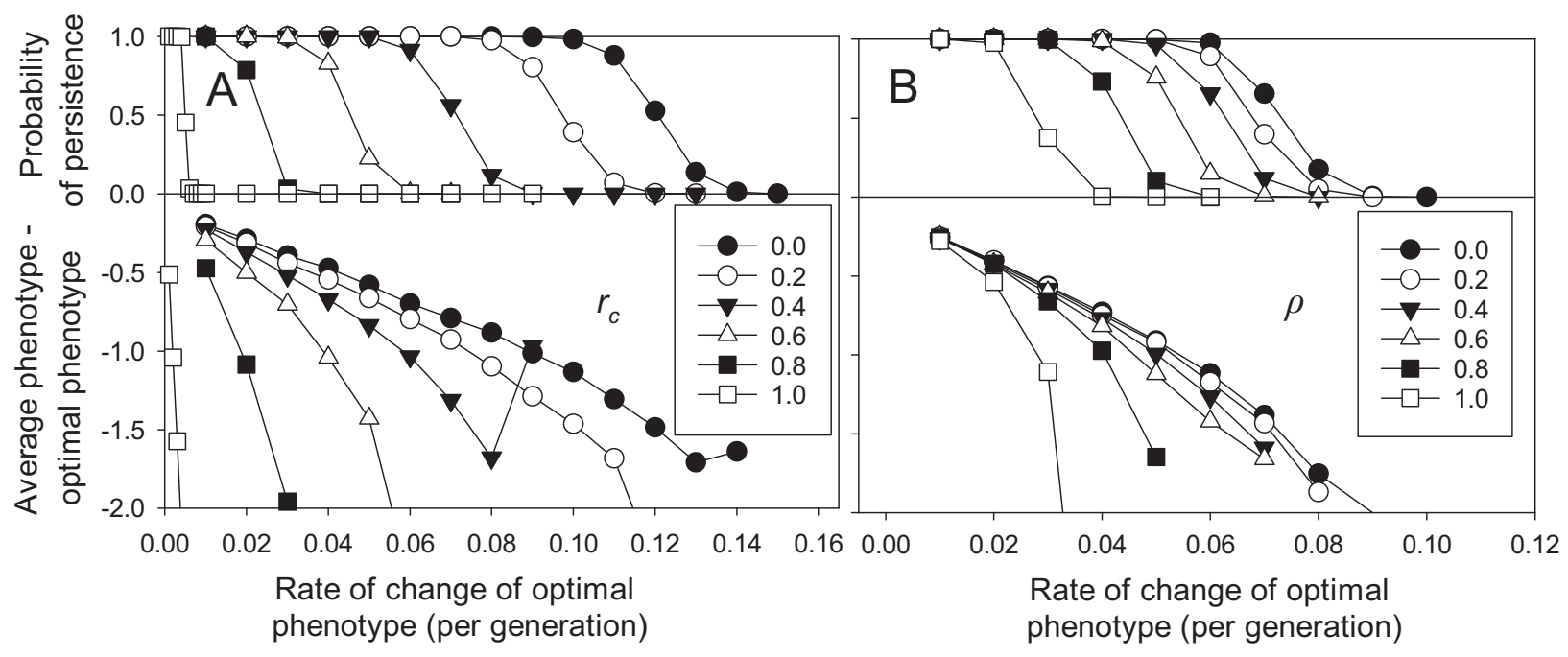

Figure 9: Probability of population persistence and evolutionary lag after a linear change in optimal phenotype with equal effort for sexual and clonal reproduction $(\delta=1)$ and $50 \%$ adult survival as a function of the rate of change, using the individual-based model with parameters as in figure 4. A, Effect of varying $r_{c}$ with $\rho=0.5$. Bffect of varying $\rho$ with $r_{c}=0.5$.

capacity. This slows selection down by, in effect, decreasing the effective population size of the component of the population that is experiencing phenotypic selection, as selection is assumed to be acting on juvenile survivorship. Moreover, under continual change adults that survive several time steps may have genotypes that were well adapted (with regard to juvenile survivorship) to past rather than present environments. This may lead to a kind of maladaptive "gene flow through time" when these adults mate at random across the population.

These effects (increased generation time and gene flow through time) do not play a role in the single-step change scenario. In contrast to a continually changing environment, a single-step change in the optimum led to a drastic reduction in population size, followed by either extinction or population growth toward the carrying capacity (results not shown for the individual-based simulations, but see figs. $2 B, 3 B$, and $3 D$ for results from the analytical model); for populations persisting but still below carrying capacity, density-independent dynamics dominated. In this case, there is no slowing of the effects of selection on juvenile survivorship. For the single-step change, increased adult survival in isolation acted to increase the overall population size during the population's decline, increasing the probability of population persistence, especially by providing more time for adaptation to occur. These simulation results mirror the analytical results discussed above for the twostage model.

This beneficial effect on population persistence after a step change was lost if an increase in adult survivorship was compensated for by a decrease in other life-history traits. If the expected rate of population decline just after the step change in the optimum is kept constant (by decreasing either the fecundity or the maximum juvenile survival as adult survival was increased), then higher adult survival leads to a lower probability of population persistence. An example for which fecundity $(f)$ was decreased as adult survival $\left(t_{22}\right)$ was increased so as to keep the initial decline at the stable stage distribution $(\lambda)$ constant is shown in figure 10 .

\section{Discussion}

Understanding the factors that promote evolutionary rescue requires one to pay attention to the "shape" of the environmental change (i.e., a single change vs. a relentless series of directional changes), to the genetic architecture underlying adaptation (a locus of major effect vs. a polygenic trait and mixtures thereof; see Gomulkiewicz et al. 2010), and to the specific life-history features of the organism experiencing the change. We have emphasized here the role that clonal reproduction may play in either facilitating or hampering evolutionary rescue, for both unstructured and structured life histories. With the analytical model, we saw dramatic effects of clonality (as measured both by the relative amounts of clonal and sexual reproduction and by the association between the environmental component of the phenotype of adults and their clonal offspring) on transients for both mean phenotype and mean genotype but not on final equilibrium values. After a sudden optimal phenotype shift, clonality slows the evolution of the genotype - and ultimately the phenotype - toward the optimum, but it can initially speed phenotypic evolution, decreasing the magni- 


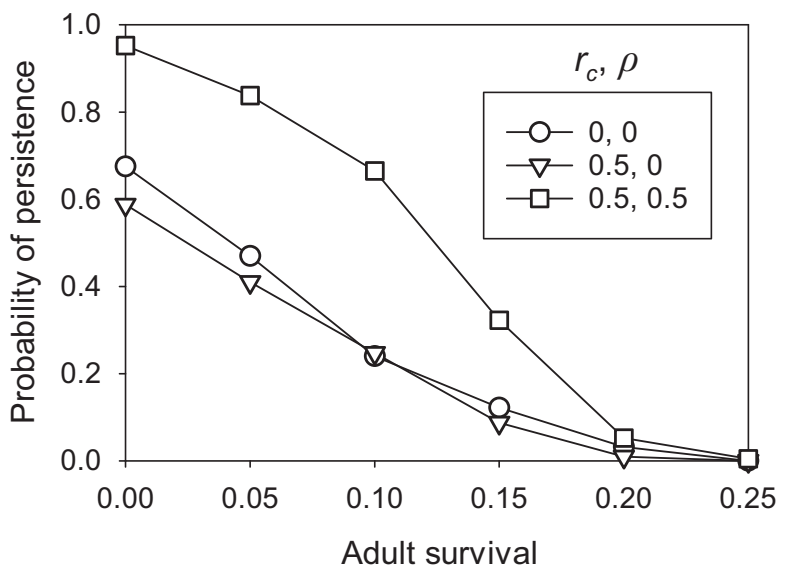

Figure 10: Probability of population persistence after a one-step change in optimal phenotype $(\theta=2.8)$ with equal effort for sexual and clonal reproduction $(\delta=1)$ as a function of adult survival, using the individual-based model with parameters $K=256, \omega^{2}=1, n=$ $10, \mu_{g}=0.001, \mu_{s}=0.00001, \alpha^{2}=0.05$, and $G=1,000$. For no adult survival, $f=4$; as adult survival was increased, $f$ was decreased to keep the expected rate of population decline just after the change in optimal phenotype constant, assuming no genetic variation.

tude and duration of the drop in population size experienced by the maladapted population, thus decreasing the probability of extinction and giving more time for evolution to take place.

The results from our individual-based simulations with both clonal and sexual reproduction show very different consequences of clonal reproduction for evolutionary lag and evolutionary rescue, depending on the type of environmental change. When considering the probability of population persistence after a one-step change, we saw greater persistence with more clonal reproduction as well as greater persistence with a higher parent-clonal offspring association, provided there was no increased cost for clonality. Evolutionary rescue here depends on the more or less immediate response of the population to its abruptly changed environment, and the variation that is exposed to selection to a first approximation will be standing, rather than novel, variation. These results support the idea that in cases in which preexisting variation is of key importance and is sufficiently available, clonal reproduction may help evolutionary rescue and concomitant phenotypic adaptation by allowing well-adapted phenotypes to avoid dissolution by sexual reproduction. In contrast, adaptation to a continually changing environment requires the constant generation of new genetic (and phenotypic) variation; allelic combinations that are currently well adapted will surely be maladapted at some future time, given a persistent directional change in the selective optimum. Here, clonal reproduction acts to decrease the availability of this needed new variation, due to the lack of recombination, a lower mutation rate for generating de novo beneficial mutations, and the parent-clonal offspring correlation in the random component of the phenotype. This same advantage of recombination and sexual reproduction for population persistence under a constantly changing environment has been observed in prior analytical and simulation models of phenotypic evolution comparing strictly sexual and strictly asexual populations rather than life histories involving both forms of reproduction, as considered here (Burger 1999; Waxman and Peck 1999).

Stage structure interacts with the type of environmental change, resulting in contrasting outcomes. In stage-structured populations with adult survival, increased adult survival acts to increase the population growth rate, providing a demographic benefit to populations far from their carrying capacities (as would be seen after a sudden step change in the optimal phenotype), thereby increasing the time available for adaptation and decreasing the probability of extinction. (This benefit is lost, however, if an increase in adult survival comes at a cost to some other life-history trait, such as juvenile survival or fecundity.) A dramatically different result is seen for a continual change in the phenotypic optimumincreased adult survival brings with it an increase in generation time that leads to more environmental change per generation, greater evolutionary lag, and a decreased probability of population persistence. An important question (beyond the scope of the current article but a target of future work) is the relative importance of clonality and other key life-history factors for population persistence. As a first step toward addressing this issue, we carried out individual-based simulations for the single-step scenario and compared the effects on population persistence of small changes in the clonal fraction, association parameter, the relative effort for clonal offspring, and adult survival (see app. B, pt. C, and fig. B2). These preliminary results indicate that while adult survival has the greatest effect on population persistence, the association parameter $(\rho)$ has nearly as great an effect, underscoring the importance of the type of clonal reproduction to our results (see below).

The few empirical and simulation studies available for the evolutionary biology of clonal organisms demonstrate that incorporation of life-history structure of this type into explicit analytical models, such as the one developed here, will be important for understanding the evolutionary potential of these populations. In a broad study of the patterns of morphological variation among clades of scleractinian reef corals, Budd (1990) found that coral lineages with greater amounts of asexual (clonal) reproduction are somewhat less variable within populations and form less morphologically discrete species than coral lineages with greater amounts of sexual reproduction, but the two groups nonetheless show generally comparable rates of speciation, extinction, and phyletic evolution. However, more highly asexual 
coral species appeared to be more susceptible to extinction associated with widespread extinction events for reef-building corals from the fossil record. Our results suggest that this outcome is particularly likely for environmental change that is both large in magnitude per generation and extends over many generations. A simulation study that considered the relative roles of mortality, dispersal ability, and age of maturation for the speed of adaptation of forest trees under climate change scenarios found that increased mortality reduced evolutionary lag significantly (Kuparinen et al. 2010). This result underscores the important point that any aspect of life history directly affecting generation time will affect evolutionary rate and therefore evolutionary lag - and one effect of clonal reproduction is to increase the overall meiotic generation time (mean time from zygote to zygote) in organisms with both sexual and asexual reproduction (Orive 1993). Our results also highlight two other effects of changing mortality. With density dependence, lower mortality for adults constrains the number of individuals exposed to selection (when selection is acting on juvenile traits, as was explored in the simulation studies here). Moreover, increased adult survivorship (decreased mortality) acts as a type of gene flow through time, where adults represent selection in past environments and so may harbor genotypes that are to a degree maladapted (when expressed as juveniles) in the current environment.

A key aspect of clonal reproduction considered in this article is its possible effect on the mapping of the nonadditive genetic and environmental components of phenotype from parent to clonal offspring. Under sexual reproduction with random mating, there is an uncoupling of the additive genetic component of phenotype from the nonadditive and environmental components. Clonal offspring, however, inherit the full parental genotype, including any dominance and epistatic (nonadditive) genetic interactions. Clonal offspring may also inherit aspects of the environmental component of phenotype, determined during development. This association $(\rho)$ between the nonadditive genetic and environmental component of parents and their clonal offspring is likely to be heavily influenced by the type of clonal reproduction involved. One can describe clonal or asexual reproduction as being either gametic (involving the products of meiosis) or agametic (involving somatic tissues; Hughes 1989). The value of the parent-clonal offspring association is likely to be 0 or close to 0 for gametic modes of clonal reproduction that most closely resemble sexual reproduction, such as the production of apomictic seeds, or at least any association would likely be comparable between sexual and gametic clonal reproduction (for a recent discussion of environmental inputs into phenotypic expression, see Sultan 2015). At the other extreme would be modes of agametic clonal reproduction involving fragmentation or fission and including a large proportion of the parental somatic tissue, where we might expect the phenotype of the clonal offspring to be extremely close to that of the parent and the association to be nearly 1 . Our simulation model assumed additive allelic effects both within and between loci, and so dominance and epistatic effects for the phenotypic trait were not considered; thus, the association considered was most closely modeling the environmental (developmental) aspects of phenotype and not the nonadditive genetic component. The analytical model, however, is general and could in the future be used to explore both the nonadditive genetic and the environmental component of clonal inheritance.

As noted earlier, use of a Gaussian fitness surface, as employed in our individual-based simulations (see app. A, pt. B), necessarily creates epistasis for fitness. Whether this epistasis is positive (and thus allelic effects on fitness are more beneficial in unison than expected from their individual effects) or negative (effects on fitness are more deleterious) depends on the magnitude of allelic effects. Generally, alleles with small beneficial fitness effects that move the mean phenotype toward the optimum on a Gaussian fitness surface will exhibit positive epistasis, while those with large effects can exhibit negative epistasis, as a combination of alleles of large effect with the same sign may cause the phenotype to overshoot the fitness optimum. Future work using the modeling framework presented here will explore the evolution of sexual versus asexual (clonal) reproduction itself; an explicit consideration of epistasis will be of key importance, as epistasis generates linkage disequilibrium (LD) of the same sign, and negative epistasis (which generates negative $\mathrm{LD}$ ) favors the evolution of recombination (and sexual reproduction; Barton 1995; Otto and Lenormand 2002). The sign of epistasis for fitness before and after environmental change may differ; how recombination affects the probability of evolutionary rescue can be shown to depend on epistasis in both phases (Uecker and Hermisson 2016).

In a study considering continuous change in strictly sexual populations, Kopp and Hermisson (2009) showed that the distribution of adaptive substitutions differed greatly depending on whether the rate of environmental change was slow relative to the "adaptive potential" of a population (in which case the adaptive process was dominated by environmental dynamics) or fast (and then the adaptive process was determined largely by genetic factors; the "mutationlimited" regime). The adaptive potential considered by Kopp and Hermisson (2009) includes both genetic factors, such as the mutation rate and the distribution of allelic effects of new mutations, and the strength of selection. This has interesting implications for our consideration of a continuously changing optimal phenotype with both sexual and clonal reproduction; we might expect, all other things being equal, that more highly sexual populations will have a greater adaptive potential and so should remain in the environmentally limited regime of the adaptive process for relatively larger rates of optimum shift (whereas the oppo- 
site should hold for more clonal populations). We plan to explore this idea in future work.

In expanding the general model of Barfield et al. (2011, 2014) for evolution in stage-structured populations to encompass clonal reproduction, we have considered a form of the clonal offspring genotype PDF, given the parental genotype, that allowed for somatic mutations to be incorporated into clonal offspring arising from somatic tissues (eq. [A5]). It was assumed that somatic mutations were not incorporated into sexual offspring. The contribution of somatic mutations to genetic load and genetic diversity at reproduction is not clear (for recent investigations, see Gross et al. 2012; Bobiwash et al. 2013); however, recent work focusing on mutation in axillary meristems in plants provides evidence that stem cell arrangement and positioning of axillary meristems may act to distribute somatic mutations around the main shoot, preventing their fixation (Burian et al. 2016). Similarly, recent work in a coral species demonstrated that while somatic mutations contributed substantially to intracolonial genetic diversity, they do not seem to transfer to gametes (Barfield et al. 2016). If somatic mutations do contribute to potentially beneficial mutations arising in sexual offspring, it would be equivalent to an overall increase in the meiotic (sexual) mutation rate for our model and so could be accounted for in that manner. We note that the work presented here does not consider unconditionally deleterious mutations; an extension allowing for such deleterious mutations as well would be an interesting avenue for future work. Moreover, our treatment of somatic mutation does not consider the action of within-individual selection. This could affect our assumption that the average genotype of clonal offspring is equal to the average genotype of their parents, since selection on somatic mutations could shift the mean clonal offspring genotype (Otto and Orive 1995; Orive 2001). This again would be an important direction for future extensions of our approach. We also did not consider phenotypic plasticity and how this additional means of response to environmental change may interact with clonal reproduction. Finally, our models are at face value applicable only to a single, spatially closed population. Extending the model to spatially structured systems with spatially varying selection (e.g., sources and sinks, as in Holt et al. 2003) would be valuable, examining, for instance, the consequences of different dispersal kernels for clonal versus sexual offspring.

A great number and variety of ecologically important organisms, including many marine invertebrates and marine and terrestrial plant species that anchor key ecosystems, have life histories with both clonal and sexual reproduction as well as stage structure. Understanding how these lifehistory characteristics combine to affect these populations' ability to respond to rapid environmental change requires the development of flexible theoretical models that account for the impact of such change on phenotypic and genotypic evolution. The approach we have presented is general for taxa with discrete life-history stages and allows explicit consideration of both sexual and clonal reproduction in the same life cycle. Our results underscore the importance of key life-history attributes, such as the degree to which clonal offspring inherit nonadditive genetic and environmental components of phenotype from their parents and the influence of stage structure on generation length, as modulators of how well species can persist in the face of different kinds of environmental change.

\section{Acknowledgments}

C.F. was supported by the Initiative for Maximizing Student Development, Office for Diversity in Science Training, and the McNair Scholars Program, University of Kansas. R.D.H. and M.B. thank the University of Florida Foundation for support. M.E.O. thanks the University of Kansas for funds in support of travel associated with this project. This work was supported by National Science Foundation grants DEB1354754 to M.E.O. and DMS-1515661/1515442 to R.D.H. We thank Y. Michalakis, S. Nuismer, J. K. Kelly, and two anonymous reviewers for careful comments and helpful suggestions that improved the manuscript.

\section{APPENDIX A}

Part A. Derivation of the General Model for StageStructured Populations with Clonal Reproduction

Recursions for the Number of Individuals and Joint PDF of Each Stage. We first consider how the number of individuals in each stage changes over time and consider three types of contributions to stage $i$ from stage $j$ : (1) transitions, where $t_{i j}(\mathbf{z})$ gives the probability that an individual with phenotype $\mathbf{z}$ in stage $j$ in the current time step directly transitions to stage $i$ in the next time step (if $i=j$, the stage does not change); (2) sexual reproduction, where $f_{i j}(\mathbf{z})$ gives the number of surviving offspring in stage $i$ produced by an individual in stage $j$ in the current time step; and (3) clonal reproduction, where $c_{i j}(\mathbf{z})$ gives the number of surviving clonal offspring in stage $i$ produced by an individual in stage $j$ in the current time step. The total contribution of each individual with phenotype $\mathbf{z}$ in stage $j$ to stage $i$ in the next time step is thus $a_{i j}(\mathbf{z})=t_{i j}(\mathbf{z})+f_{i j}(\mathbf{z})+c_{i j}(\mathbf{z})$. Note that $1-\sum_{i} t_{i j}(\mathbf{z})$ is the probability of death of a stage $j$ individual, since it is the probability that it does not transition to any stage. For each pair of stages $i$ and $j$, we can write the average transition probability from stage $j$ to stage $i, \bar{t}_{i j}$, and the average number of sexual offspring, $\bar{f}_{i j}$, and clonal offspring, $\bar{c}_{i j}$, produced in stage $i$ from each stage $j$ individual as 


$$
\begin{aligned}
& \bar{t}_{i j}=\iint t_{i j}(\mathbf{z}) p_{j}(\mathbf{g}, \mathbf{z}) d \mathbf{z} d \mathbf{g}, \\
& \bar{f}_{i j}=\iint f_{i j}(\mathbf{z}) p_{j}(\mathbf{g}, \mathbf{z}) d \mathbf{z} d \mathbf{g}, \\
& \bar{c}_{i j}=\iint c_{i j}(\mathbf{z}) p_{j}(\mathbf{g}, \mathbf{z}) d \mathbf{z} d \mathbf{g}, \\
& \bar{a}_{i j}=\iint a_{i j}(\mathbf{z}) p_{j}(\mathbf{g}, \mathbf{z}) d \mathbf{z} d \mathbf{g}=\bar{t}_{i j}+\bar{f}_{i j}+\bar{c}_{i j},
\end{aligned}
$$

where $p_{j}(\mathbf{g}, \mathbf{z})$ is the current joint PDF of genotype $\mathbf{g}$ and phenotype $\mathbf{z}$ in stage $j$ and the integrals are evaluated over all values of $\mathbf{g}$ and $\mathbf{z}$. This lets us write the population size recursion for stage $i$ as

$$
N_{i}^{\prime}=\sum_{j} N_{j} \bar{t}_{i j}+\sum_{j} N_{j} \bar{f}_{i j}+\sum_{j} N_{j} \bar{c}_{i j}=T_{i}^{\prime}+F_{i}^{\prime}+C_{i}^{\prime},
$$

where $T_{i}^{\prime}=\sum_{j} N_{j} \bar{t}_{i j}, F_{i}^{\prime}=\sum_{j} N_{j} \bar{f}_{i j}$, and $C_{i}^{\prime}=\sum_{j} N_{j} \bar{c}_{i j}$ (and $\left.N_{i}^{\prime}=\sum_{j} N_{j} \bar{a}_{i j}\right)$.

Next, consider the recursion for the joint PDF of $\mathbf{g}$ and $\mathbf{z}$. Let $p_{i}^{\prime}(\mathbf{g}, \mathbf{z})$ be the joint PDF in the next time step, which can be written as

$$
p_{i}^{\prime}(\mathbf{g}, \mathbf{z})=\theta_{i}(\mathbf{g}, \mathbf{z}) \frac{T_{i}^{\prime}}{N_{i}^{\prime}}+\phi_{i}(\mathbf{g}, \mathbf{z}) \frac{F_{i}^{\prime}}{N_{i}^{\prime}}+\kappa_{i}(\mathbf{g}, \mathbf{z}) \frac{C_{i}^{\prime}}{N_{i}^{\prime}},
$$

where $\theta_{i}(\mathbf{g}, \mathbf{z})=\sum_{j} N_{j} t_{i j}(\mathbf{z}) p_{j}(\mathbf{g}, \mathbf{z}) / T_{i}^{\prime}$ is the joint PDF of all individuals that transition to stage $i$ and $\phi_{i}(\mathbf{g}, \mathbf{z})$ is the joint PDF of sexual offspring into stage $i$, which depends on the details of reproduction. (Note that eqq. [A1]-[A3] are analogous to eqq. [1]-[4] in Barfield et al. [2011], and an example of $\phi_{i}(\mathbf{g}, \mathbf{z})$ is derived in app. C there under the assumption of random mating and an infinitesimal model of inheritance.)

We then need to derive the joint distribution of $\mathbf{g}$ and $\mathbf{z}$ for clonal offspring into stage $i, \kappa_{i}(\mathbf{g}, \mathbf{z})$. This joint PDF can be written as

$$
\begin{aligned}
\kappa_{i}(\mathbf{g}, \mathbf{z}) & =\sum_{j}\left[\frac{1}{\bar{c}_{i j}} \iint S_{i j}\left(\mathbf{g}, \mathbf{z} \mid \mathbf{g}^{*}, \mathbf{z}^{*}\right) c_{i j}\left(\mathbf{z}^{*}\right) p_{j}\left(\mathbf{g}^{*}, \mathbf{z}^{*}\right) d \mathbf{g}^{*} d \mathbf{z}^{*}\right]\left[\frac{\bar{c}_{i j} N_{j}}{C_{i}^{\prime}}\right] \\
& =\frac{1}{C_{i}^{\prime}} \sum_{j} N_{j} \iint S_{i j}\left(\mathbf{g}, \mathbf{z} \mid \mathbf{g}^{*}, \mathbf{z}^{*}\right) c_{i j}\left(\mathbf{z}^{*}\right) p_{j}\left(\mathbf{g}^{*}, \mathbf{z}^{*}\right) d \mathbf{g}^{*} d \mathbf{z}^{*},
\end{aligned}
$$

where the function $S_{i j}\left(\mathbf{g}, \mathbf{z} \mid \mathbf{g}^{*}, \mathbf{z}^{*}\right)$ gives the joint PDF (of genotype $\mathbf{g}$ and phenotype $\mathbf{z}$ ) of clonal offspring in stage $i$ with stage $j$ parents with genotype $\mathbf{g}^{*}$ and phenotype $\mathbf{z}^{*}$. The first term in brackets in the first expression is the joint PDF of clonal offspring into stage $i$ with parents in stage $j$ (averaging the $S_{i j}$ function, weighted by the clonal fecundities, over parental genotypes and phenotypes). The second term in brackets is the fraction of clonal offspring into stage $i$ contributed by parents in stage $j$; this factor weights the corresponding joint PDF in the first term.

The $S_{i j}$ function in equation (A4) can be separated into two probability functions, one for the offspring genotype and one for its phenotype (given its genotype), so that $S_{i j}\left(\mathbf{g}, \mathbf{z} \mid \mathbf{g}^{*}, \mathbf{z}^{*}\right)=X_{i j}\left(\mathbf{g} \mid \mathbf{g}^{*}, \mathbf{z}^{*}\right) Y_{i j}\left(\mathbf{z} \mid \mathbf{g}, \mathbf{g}^{*}, \mathbf{z}^{*}\right)$. One can often reasonably assume that $X_{i j}\left(\mathbf{g} \mid \mathbf{g}^{*}, \mathbf{z}^{*}\right)=X_{i j}\left(\mathbf{g} \mid \mathbf{g}^{*}\right)$, since offspring genotype will not depend on the parental phenotype in the absence of any transgenerational epigenetic effects. (For a recent discussion of the possible importance of transgenerational epigenetic effects, see Grossniklaus et al. 2013; Sultan 2015.)

Examples of Clonal Offspring Joint PDF Given Parental Joint PDF. In the absence of somatic mutation, the genotypic distribution of clonal offspring would be unchanged from that of their parents. However, since some forms of clonal reproduction can allow somatic mutations to be incorporated into the clonal offspring, we allow somatic mutation to change the distribution. Somatic mutation can be modeled as an additive value to genotype, so that offspring genotype $\mathbf{g}=\mathbf{g}^{*}+\mathbf{m}$, where $\mathbf{g}^{*}$ is the parental genotype and mutation effect $\mathbf{m}$ has a multivariate Gaussian distribution with mean $\mathbf{0}$ and covariance matrix $\mathbf{V}_{\mathrm{M}}$. The distribution of clonal offspring genotype, given the genotype of the parent, is then

$$
\begin{gathered}
X_{i j}\left(\mathbf{g} \mid \mathbf{g}^{*}\right)= \\
\frac{1}{\sqrt{(2 \pi)^{m} \operatorname{det}\left(\mathbf{V}_{\mathrm{M}, i j}\right)}} \exp \left[-\frac{1}{2}\left(\mathbf{g}-\mathbf{g}^{*}\right)^{\mathrm{T}} \mathbf{V}_{\mathrm{M}, i j}^{-1}\left(\mathbf{g}-\mathbf{g}^{*}\right)\right],
\end{gathered}
$$

where the subscript $i j$ on $\mathbf{V}_{\mathrm{M}}$ indicates that the variances and covariances for the somatic mutation process may depend on the specific offspring and parent stages, and again $m$ gives the number of components for the multivariate phenotype.

The next step is to consider the PDF of phenotype (given genotype) for the clonal offspring, $Y_{i j}\left(\mathbf{z} \mid \mathbf{g}, \mathbf{g}^{*}, \mathbf{z}^{*}\right)$. We might consider this to be the same as for sexual offspring, giving

$$
\begin{gathered}
Y_{i j}\left(\mathbf{z} \mid \mathbf{g}, \mathbf{g}^{*}, \mathbf{z}^{*}\right)= \\
\exp \left[-\frac{1}{2}(\mathbf{z}-\mathbf{g})^{\mathrm{T}} \mathbf{V}_{\mathrm{E}}^{-1}(\mathbf{z}-\mathbf{g})\right] / \sqrt{(2 \pi)^{m} \operatorname{det}\left(\mathbf{V}_{\mathrm{E}}\right)}
\end{gathered}
$$

where $\mathbf{V}_{\mathrm{E}}$ is the covariance matrix for the random part of the phenotype. However, clonal reproduction may affect the mapping of $\mathbf{g}$ and $\mathbf{e}$ onto phenotype.

Therefore, we define an $m$-dimensional vector of association parameters, $\boldsymbol{\rho}_{i j}$, which gives the association between e for a stage $i$ clonal offspring and $\mathbf{e}^{*}$ for its stage $j$ parent. Thus, $\rho_{i j k}$ gives this association for the $k$ th component of the multivariate trait $\left(z_{k}\right)$. One possible assumption is that each offspring component of $\mathbf{e}$ is generated by taking a 
weighted sum of the corresponding parental component and a random portion. For example, for $z_{k}$ we could have

$$
e_{k}=\rho_{i j k} e_{k}^{*}+\sqrt{\left(1-\rho_{i j k}^{2}\right) V_{E, i j k}} \zeta,
$$

where $e_{k}$ is the environmental part of the phenotype of a stage $i$ clonal offspring, $e_{k}^{*}$ is that of its stage $j$ parent, and $\zeta$ is a zero-mean, unit-variance Gaussian deviate. Thus, if $\rho_{i j k}$ is 0 , the offspring environmental value is completely randomly determined and has a zero-mean Gaussian distribution with variance $V_{E, i j k}$. If $\rho_{i j k}=1$, the environmental value of the parent is passed on exactly to its offspring. Other values of $\rho_{i j k}$ (between 0 and 1) result in the environmental part of clonal offspring phenotype being partly random and partly determined by the parental value. We note that the association parameter will be the correlation coefficient if the environmental value of the parent has a mean equal to 0 and variance equal to $V_{E, i j k}$. However, more generally this parameter is not a proper correlation coefficient.

With this assumption for all components of $\mathbf{e}$, the PDF of $z_{k}$ given $g_{k}, z_{k}^{*}$, and $g_{k}^{*}$ is Gaussian with mean $g_{k}+$ $\rho_{i j k}\left(z_{k}^{*}-g_{k}^{*}\right)$ and variance $\left(1-\rho_{i j k}^{2}\right) V_{E, i j k}$, and the corresponding joint PDF is the product of these PDFs over all $k$. This joint PDF is $Y_{i j}\left(\mathbf{z} \mid \mathbf{g}, \mathbf{g}^{*}, \mathbf{z}^{*}\right)$, and its average value is

$$
\int \mathbf{z} Y_{i j}\left(\mathbf{z} \mid \mathbf{g}, \mathbf{g}^{*}, \mathbf{z}^{*}\right) d \mathbf{z}=\mathbf{g}+\mathbf{R}_{i j}\left(\mathbf{z}^{*}-\mathbf{g}^{*}\right),
$$

where $\mathbf{R}_{i j}$ is the diagonal matrix with elements $\rho_{i j k}$. (The right side of eq. [A7] is just a vector with elements $g_{k}+\rho_{i j k}\left(z_{k}^{*}-\right.$ $g_{k}^{*}$ ), the means of $z_{k}$ given $g_{k}, z_{k}^{*}$, and $g_{k}^{*}$.) Note that this formulation assumes that the environmental part of each component of the offspring phenotype is correlated only with the environmental part of the same component of the parent's phenotype.

\section{Part B. Individual-Based Simulations}

We used an individual-based model (IBM) to simulate the effects of clonality on environmental rescue in response to an environmental change that is either sudden (a step change) or gradual (a linear change). The IBM explicitly tracks each individual and its genotype; it complements the deterministic treatment of selection in the main body of the article and appendix A, part A, by including realistic features of evolution in natural populations, such as mutation, drift, and demographic stochasticity (which permits extinction). The assumptions of the IBM are based on the assumptions made in an IBM explored by Burger and Lynch (1995). We have modified this model in the current article to allow some (or all) offspring to be produced clonally, as well as in other ways (see details below). Individuals are assumed to be diploid and hermaphroditic. For the Burger and Lynch (1995) model and many of the results here, adults die after reproduction, so there are nonoverlapping generations. However, in some cases we allow adults to survive for multiple time periods, with a fixed probability of survival for each period. We refer to the time period as a year, which is the generation time if there is no adult survival.

Each individual has a one-dimensional trait (phenotype) that determines its probability of survival from juvenile to adult (the life-history transition when selection takes place: $t$ for the one-stage model, and $t_{21}$ for the two-stage model with adult survival; see fig. 1). We note that this is only one of several feasible selection schemata contained in the general analytical machinery explored in the main text. This survival probability is a Gaussian function of the difference between the individual's phenotype and an optimal phenotype for the environment and is given by $W_{k t}=$ $\exp \left\{-\left(z_{k t}-z_{\text {opt }, t}\right)^{2} /\left(2 \omega^{2}\right)\right\}$, where $W_{k t}$ is the probability of survival from juvenile to adult for the $k$ th juvenile in year $t, z_{k t}$ is its phenotype, $z_{\mathrm{opt}, t}$ is the optimal phenotype for that year, and $\omega$ determines the strength of selection on phenotype (a lower value being stronger selection).

Density regulation follows selection. Density is regulated above a population size "ceiling" by limiting the number of mating sites to a value $K$. If there are fewer than $K$ adults, then all adults mate as a female, while if there are more than $K$ adults, then $K$ are chosen at random (without replacement) to act as reproductive females (this mating system thus differs from that in Burger and Lynch [1995] and Holt et al. [2003]). Each mating female mates with a randomly selected (with replacement) adult acting as male, which can be the same individual as the female (so selfing is allowed). If there is no adult survival (as assumed in most of our simulations), then the adult pool each year consists of only those juveniles that survived selection in that year. In simulations with adult survival, in addition to surviving juveniles the adult pool also contains adult survivors from the previous year; in this case, each adult mating as a female survives until the next year with probability $p_{s}$ (equivalent to $t_{22}$ in the analytical model). The mating adults are chosen at random from this pool, with new adults and adult survivors from the previous year equally likely to be chosen.

Each mating pair then produces offspring. Offspring can be produced either sexually or clonally. Our measure of total reproductive effort is the number of sexual offspring that would be produced were all offspring sexual (the average reproductive effort per reproducing female is denoted by $f$ ). The amount of clonal reproduction is given as the fraction of reproductive effort that is devoted to clonal reproduction $\left(r_{c}\right)$. Clonal offspring may require more resources to produce, so the cost of producing a clonal offspring (relative to a sexual offspring) must be specified $(\delta)$. If $\delta=1$, the effort required to produce a clonal offspring is the same as that for a sexual offspring, and $r_{c}$ is the probability that each offspring is clonal. If $\delta>1$, then clonal offspring require more effort, so fewer clonal offspring are produced (and fewer to- 
tal offspring), so that the reproductive effort devoted to clonal offspring is the specified value of $r_{c}$ (e.g., for $r_{c}=$ 0.5 with $\delta=1.5$, there would be $f / 2$ sexual offspring and $f / 3$ clonal offspring, so that sexual and clonal reproduction each represent $50 \%$ of the total reproductive effort, $f$ ). We did not consider cases in which clonal offspring are less costly than sexual offspring $(\delta<1)$, nor did we include the classic "cost of sex" (the cost of males or the cost of male function in hermaphrodites; Maynard Smith 1978). If we had done so, it would have given an additional advantage to clonality under a step change and an additional disadvantage to clonality under continual change (results not shown). Finally, we note that these measures of reproduction can also implicitly include components of juvenile survivorship up to the point when selection acts on the trait and are thus effective reproduction rates, giving the number of offspring that survive to this point.

For most simulations, the average number of offspring per reproducing female was an integer, in which case each female produced exactly that number (but the number of clonal and sexual offspring was still random; if the parameters called for each female to produce two clonal and two sexual offspring, each female produced four offspring, each of which was clonal with probability 0.5 ). For a few simulations, the number of offspring was not an integer, so the number produced per female was a random variable with the required mean. This often occurred when the relative effort for clonal offspring was 1.5. For the example discussed above, with $50 \%$ clonality and $\delta=1.5$, the number of clonal offspring was $f / 3$, and we generally used $f=4$, which gave $4 / 3$ clonal offspring per female (and two clonal offspring). In these cases, the number of potential offspring was the first integer greater than or equal to the total average offspring (in this case, the total is 3.3333, so four was used). The fate of each potential offspring was then determined using the appropriate probability (in this case, for each potential offspring the probability was $1 / 2$ that it was sexual, $1 / 3$ that it was clonal, and $1 / 6$ that it was not produced). The other situation in which the number of offspring was not an integer was when the adult survivorship was varied and the fecundity adjusted to give the same initial rate of population decline. In this case, the number of potential offspring was fixed at four, and the decrease in fecundity with increasing adult survival was achieved by decreasing the probabilities associated with each type of offspring.

The general machinery developed in the main text, in part A of appendix A, and in part A of appendix B describes the evolution of multivariate traits, but in our simulation models reported here we focus on a single trait experiencing selection. An individual's phenotype is the sum of allelic values (which can be any real numbers) at $n$ diploid loci and a random component. In the results reported here, we set $n=10$; in past studies, we have found little effect of variation in the number of loci if $n>5$ (Holt et al. 2003; M. Barfield and R. D. Holt, unpublished results). During reproduction, there is free recombination between all loci for sexual reproduction, with no dominance or epistasis. For clonal reproduction, both haplotypes of the parent (the female in the mating pair) are passed to the offspring, so linkage is complete. For sexual reproduction, each offspring haplotype then mutates with probability $n \mu_{g}$ (here, $\mu_{g}$ is the per locus gametic or sexual mutation rate); if a mutation occurs, a random locus is selected, and a zero-mean Gaussian deviate with variance $\alpha^{2}$ is added to the previous allelic value. For clonal reproduction, the mutation procedure is the same, but a different, generally lower per-locus mutation rate, $\mu_{s}$, may be used (simulating somatic mutation). After the alleles of each offspring are determined, the random part of its phenotype is chosen. For sexual reproduction, the random part of phenotype was an independent deviate from a zero-mean, unit-variance Gaussian distribution. For clonal reproduction, the offspring's random value could be influenced by its parent's value through an association parameter $\rho$ (see the main text for further explanation). The random part of the offspring's phenotype is determined using the expression

$$
e_{k}=\rho e_{k}^{*}+\sqrt{1-\rho^{2}} \zeta,
$$

where $e_{k}$ and $e_{k}^{*}$ are, respectively, the random parts of the offspring's and parent's phenotypes and $\zeta$ is a zero-mean, unit-variance Gaussian random deviate (for details, see app. A, pt. A).

For all simulations reported here, a population was initiated with $K$ adults with random allelic values. One thousand time steps were then simulated with the optimal phenotype constant at 0 . This protocol allows the population to reach mutation-selection-drift equilibrium, with a standing amount of genetic variation maintained by an input of mutational variation and depleted by both stabilizing selection and drift. The genetics of the population at the end of this period should be independent of the allelic values of the initial population, which were chosen to give a reasonable initial genetic variance so that this equilibration would occur faster; the procedure used to assign these initial alleles is that described in Burger and Lynch (1995). Carrying capacities and fecundities were chosen so that extinction due to demographic stochasticity in the constant environment was extremely unlikely (and indeed never observed in our simulation runs). Unless otherwise indicated, reproduction was completely sexual during this equilibration period, which ensures that all populations have the same expected genetic variance at the time of the environmental change.

After the equilibration period, in the case of the step environmental change the optimal phenotype was changed suddenly to a positive value, $\theta$. In the case of the linear (continuous) environmental change, the optimal phenotype was 
proportional to the number of generations since the environment began to change. Each simulation was then continued for another $G$ years. Many simulations (generally 400 replicates) were performed for each set of parameters, and the probability of persistence was estimated as the fraction of such populations that were not extinct after this $G$-year period. Parameters maintained constant across simulations were $K=256, f=4$ (except for fig. 10 ), $\omega^{2}=1$, $n=10, \mu_{g}=0.001, \alpha^{2}=0.05$, and $G=1,000$. The somatic mutation rate was $\mu_{s}=0.00001$ unless otherwise indicated, reflecting the expected lower rate of somatic (mitotic) mutation than gametic (meiotic) mutation (see the main text). In one case, we used $\mu_{s}=0.001$ to equalize mutational input for sexual and clonal reproduction. For each type of environmental change, we varied the clonal fraction of reproductive effort $r_{c}$, the association parameter $\rho$, the relative effort for clonal reproduction $\delta$, and the degree of change of the environment $(\theta$ for the step change, the rate of change for the linear change). For each parameter set we recorded the probability of population persistence, and for the linear environmental change we also recorded the difference between the population average phenotype and the optimal phenotype for that year, averaged over the last 200 years for all populations that survived for the entire simulation. This measure characterizes the phenotypic lag, which in turn is related to the degree of maladaptation expected in populations persisting in these continually changing environments.

\section{Literature Cited}

Barfield, M., R. D. Holt, and R. Gomulkiewicz. 2011. Evolution in stage-structured populations. American Naturalist 177:397-409.

. 2014. Correction. American Naturalist 184:284-287.

Barfield, S., G. V. Aglyamova, and M. V. Matz. 2016. Evolutionary origins of germline segregation in Metazoa: evidence for a germ stem cell lineage in the coral Orbicella faveolata (Cnidaria, Anthozoa) Proceedings of the Roval Societv B 283:20152128.

Barton, N. H. 1995. A general model for the evolution of recombination. Genetical Research 65:123-144.

Barton, N. H., and L. Partridge. 2000. Limits to natural selection. BioEssavs 22:1075-1084.

Bell, G. 2013. Evolutionary rescue and the limits of adaptation. Philosophical Transactions of the Roval Societv B 368:20120080.

Bobiwash, K., S. T. Schultz, and D. J. Schoen. 2013. Somatic deleterious mutation rate in a woody plant: estimation from phenotypic data. Heredity 111:338-344.

Budd, A. F. 1990. Longterm patterns of morphological variation within and among species of reef-corals and their relationship to sexual reproduction. Systematic Botany 15:150-165.

Burian, A., P. B. de Reuille, and C. Kuhlemeier. 2016. Patterns of stem cell divisions contribute to plant longevity. Current Biology 26:1-10.

Burger, R. 1999. Evolution of genetic variability and the advantage of sex and recombination in changing environments. Genetics 153:10551069.
Burger, R., and M. Lynch. 1995. Evolution and extinction in a changing environment: a quantitative-genetic analysis. Evolution 49:151-163.

Crespi, B. J. 2000. The evolution of maladaptation. Heredity 84:623639.

Gandon, S., M. E. Hochberg, R. D. Holt, and T. Day. 2013. What limits the evolutionary emergence of pathogens? Philosophical Transactions of the Roval Societv B 368:20120086.

Gomulkiewicz, R., and R. D. Holt. 1995. When does evolution by natural selection prevent extinction? Evolution 49:201-207.

Gomulkiewicz, R., R. D. Holt, M. Barfield, and S. L. Nuismer. 2010. Genetics, adaptation, and invasion in harsh environments. Evolutionary Applications 3:97-108.

Gomulkiewicz, R., and D. Houle. 2009. Demographic and genetic constraints on evolution. American Naturalist 174:E218-E229.

Gonzalez, A., and G. Bell. 2013. Evolutionary rescue and adaptation to abrupt environmental change depends upon the history of stress. Philosophical Proceedings of the Roval Society B 368:20120079.

Gonzalez, A., O. Ronce, R. Ferriere, and M. E. Hochberg. 2013. Evolutionary rescue: an emerging focus at the intersection between ecology and evolution. Philosophical Proceedings of the Roval Societv B 368:20120404.

Gross, C. L., P. A. Nelson, A. Haddadchi, and M. Fatemi. 2012. Somatic mutations contribute to genotypic diversity in sterile and fertile populations of the threatened shrub, Grevillea rhizomatosa (Proteaceae). Annals of Botany 109:331-342.

Grossniklaus, U., W. G. Kelly, A. C. Ferguson-Smith, M. Pembrey, and S. Lindquist. 2013. Transgenerational epigenetic inheritance: how important is it? Nature Reviews Genetics 14:228-235.

Herrera, C. M. 2009. Multiplicity in unity: plant subindividual variation and interactions with animals. University of Chicago Press, Chicago.

Hoffmann, A. A., and C. M. Sgrò. 2011. Climate change and evolutionary adaptation. Nature 470:479-485.

Holt, R. D. 1990. The microevolutionary consequences of climate change. Trends in Ecology and Evolution 5:311-315.

Holt, R. D., M. Barfield, and R. Gomulkiewicz. 2005. Theories of niche conservatism and evolution: could exotic species be potential tests? Pages 259-290 in D. Sax, J. Stachowicz, and S. D. Gaines, eds. Species invasions: insights into ecology, evolution, and biogeography. Sinauer, Sunderland, MA.

Holt, R. D., R. Gomulkiewicz, and M. Barfield. 2003. The phenomenology of niche evolution via quantitative traits in a 'black-hole' sink. Proceedings of the Roval Societv B 270:215-224.

Hughes, R. N. 1989. A functional biology of clonal animals. Chapman \& Hall, London.

Jackson, J. B. C., L. W. Buss, and R. E. Cook, eds. 1985. Population biology and evolution of clonal organisms. Yale University Press, New Haven, CT.

Kinnison, M. T., N. G. Hairston Jr., and A. P. Hendry. 2015. Cryptic eco-evolutionary dynamics. Annals of the New York Academy of Sciences 1360:120-125.

Kopp, M., and J. Hermisson. 2009. The genetic basis of phenotypic adaptation. II. The distribution of adaptive substitutions in the moving optimum model. Genetics 183:1453-1476.

Kuparinen, A., O. Savolainen, and F. M. Schurr. 2010. Increased mortality can promote evolutionary adaptation of forest trees to climate change. Forest Ecology and Management 259:1003-1008.

Lindgren, D. 1975. Sensitivity of premeiotic and meiotic stages to spontaneous and induced mutations in barley and maize. Hereditas 79:227-238. 
Magni, G. E., and R. C. Von Borstel. 1962. Different rates of spontaneous mutation during mitosis and meiosis in yeast. Genetics 47:10971108 .

Martin, G., R. Aguilee, J. Ramsayer, O. Kaltz, and O. Ronce. 2013. The probability of evolutionary rescue: towards a quantitative comparison between theory and evolution experiments. Philosophical Transactions of the Roval Societv B 368:20120088.

Maynard Smith, J. 1976a. A comment on the Red Queen. American Naturalist 110:325-330.

. 1976b. What determines the rate of evolution? American Naturalist 110:331-338.

. 1978. The evolution of sex. Cambridge University Press, Cambridge.

Orive, M. E. 1993. Effective population size in organisms with complex life histories. Theoretical Population Biology 44:316-340. 1995. Senescence in organisms with clonal reproduction and complex life histories. American Naturalist 145:90-108.

2001. Somatic mutations in organisms with complex life histories. Theoretical Population Biology 59:235-249.

Orr, H. A., and R. L. Unckless. 2008. Population extinction and the genetics of adaptation. American Naturalist 172:160-169.

2014. The population genetics of evolutionary rescue. PLoS Genetics 10:e1004551.

Otto, S. P., and T. Lenormand. 2002. Resolving the paradox of sex and recombination. Nature Review Genetics 3:252-261.
Otto, S. P., and M. E. Orive. 1995. Evolutionary consequences of mutation and selection within an individual. Genetics 141:1173-1187.

Pandolfi, J. M., S. R. Connolly, D. J. Marshall, and A. L. Cohen. 2011. Projecting coral reef futures under global warming and ocean acidification. Science 333:418-422.

Schiffers, K., E. C. Bourne, S. Lavergne, W. Thuiller, and J. M. J. Travis. 2013. Limited evolutionary rescue of locally adapted populations facing climate change. Philosophical Transactions of the Roval Societv B 368:20120083.

Sultan, S. E. 2015. Organism and environment: ecological development, niche construction, and adaptation. Oxford University Press, Oxford.

Rattray, A., G. Santoyo, B. Shafer, and J. N. Strathern. 2015. Elevated mutation rate during meiosis in Saccharomyces cerevisiae. PLoS Genetics 11:e1004910.

Uecker, H., and J. Hermisson. 2016. The role of recombination in evolutionary rescue. Genetics 202:721-732.

Uecker, H., S. P. Otto, and J. Hermisson. 2014. Evolutionary rescue in structured populations. American Naturalist 183:E17-E35.

Waxman, E., and J. R. Peck. 1999. Sex and adaptation in a changing environment. Genetics 153:1041-1053.

Wilson, E. O. 2016. Half-earth: our planet's fight for life. Norton, New York.

Associate Editor: Scott L. Nuismer Editor: Yannis Michalakis

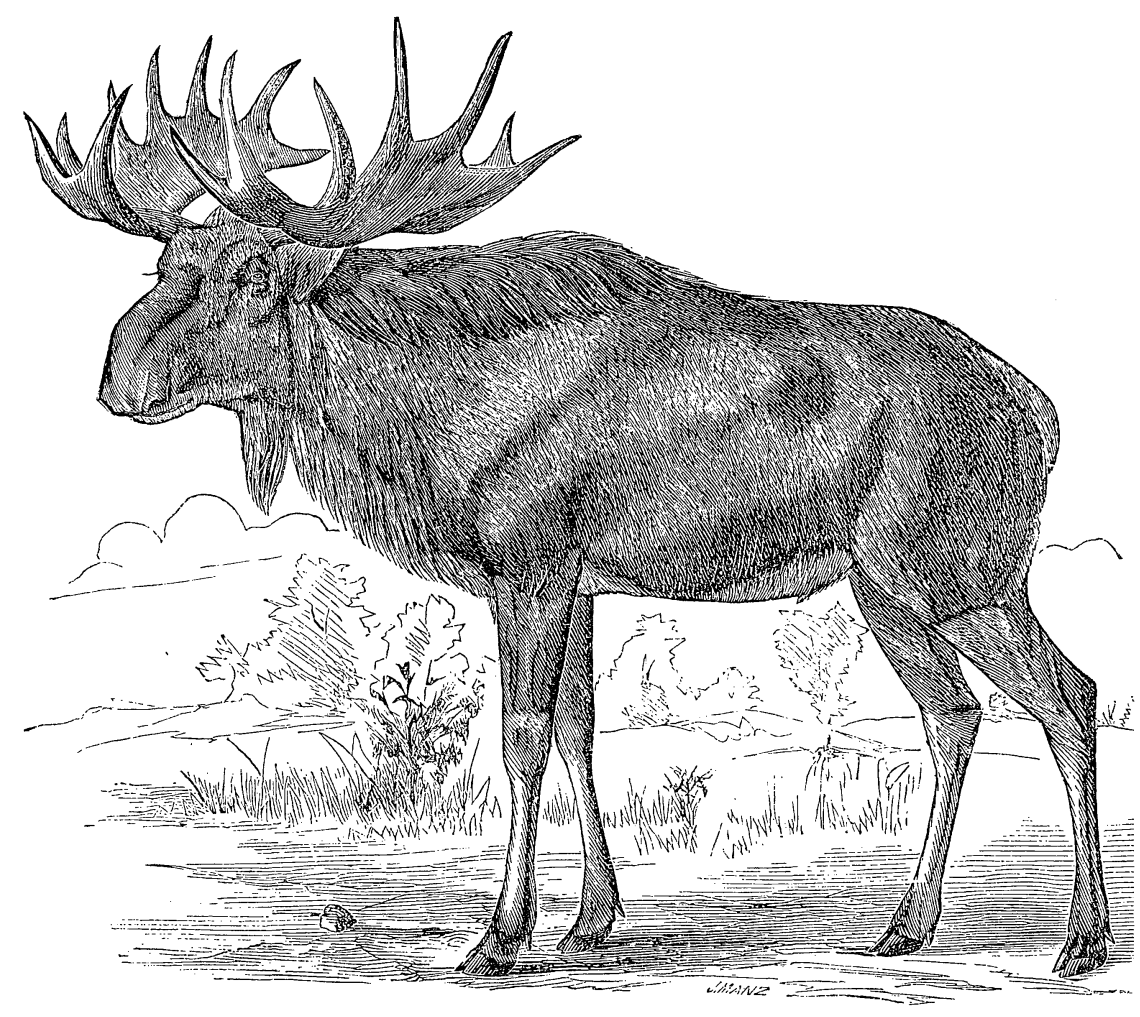

"Judge Caton is one of those pleasant, persuasive writers who will take no denial; he carries us along whether we will or not, and ends by making us wonder why everybody does not turn to keeping deer and studying their structure and habits!" Figured: Scandinavian elk. From the review of Caton's Deer of America (The American Naturalist, 1877, 11:354-357). 